\title{
A phase field model for the growth and characteristic thickness of deformation-induced twins
}

\author{
Nicolò Grilli ${ }^{\mathrm{a}, *}$, Alan C.F. Cocks ${ }^{\mathrm{a}}$, Edmund Tarleton ${ }^{\mathrm{a}, \mathrm{b}}$ \\ ${ }^{a}$ Department of Engineering Science, University of Oxford, Parks Road, OX1 3PJ, UK \\ ${ }^{b}$ Department of Materials, University of Oxford, Parks Road, OX1 3PH, UK
}

\begin{abstract}
Deformation-induced twinning is an important mechanism in metals with a limited number of slip deformation modes. The mechanisms for twin nucleation and growth are not completely understood, and modelling these processes is challenging because of the different length and time scales involved. Twins grow at the speed of sound up to a length of several millimetres and thickness of only a few microns. We present a phase field model for twinning, coupled with a dislocation-density based model for slip, implemented within the crystal plasticity finite element method. Softening of the critical resolved shear stress for twinning is used to reproduce the shear localisation that is typical of twin bands. Two interaction terms are introduced. The first one is a non-local term that models the interaction between residual dislocations at the twin interface and mobile dislocations in untwinned regions. The second is a local term that models the hardening of the twin system due to the presence of dislocations. By introducing these interaction terms, it is possible to reproduce a discrete pattern of twin bands after deformation. These interaction terms and interaction strength parameters determine the nucleation and spatial position of twins, twin thickness and number density of twins as a function of strain. The model is validated by comparing the simulated twin phase field with the dynamic formation of twins in tension, as measured by electron backscatter diffraction experiments on $\alpha$-uranium. This model sheds light on the mechanism that determines twin growth and twin thickness. Specifically, twins stop thickening after a critical density of residual dislocations at the twin interface is reached. The interaction coefficients are interpreted in terms of the stacking fault energy in order to apply the model to different metals. ${ }^{1}$
\end{abstract}

Keywords: Crystal Plasticity, Dislocations, Twinning, Tensile test, EBSD, Uranium

\section{Introduction}

Twinning is an important deformation mechanism in crystalline materials [1], especially those with hexagonal and orthorhombic [2] crystal structures. Because of the atomic rearrangement, in

\footnotetext{
${ }^{1}$ Published version available at 10.1016/j.jmps.2020.104061

*Corresponding author

Email address: nicolo.grilli@eng.ox.ac.uk (Nicolò Grilli)
} 
which atoms form a mirror image of the original crystal in a narrow band, plastic strain localises inside the twins [3]. This localisation leads to high stress at the intersection between twin bands and grain boundaries [4], which can initiate microcracks [5]. Twin interfaces represent barriers for dislocation motion, therefore they produce a size effect similar to grain boundaries [6], i.e. additional hardening of the slip systems that depends on the spacing between twin bands [7]. This is particularly important because of recent experiments showing that nanotwinned materials with high strength and ductility can be manufactured $[8,9]$. Modelling the nucleation and evolution of strain-induced twin bands is an open problem. The solution would be important for materials engineering because the effect of texture, microstructure and alloying elements on strain-induced twins could be predicted.

Twin nucleation is linked to the formation of multiple stacking faults and to the lack of dislocation slip systems. The stacking fault energy (SFE) and $\gamma$ energy surface, which is the extra crystal energy as a function of the translation vector when an atomic plane slides over another one, determine if twin nucleation is favoured [10]. Twin nucleation mechanisms have been proposed for different crystal structures based on atomistic simulations: a pole mechanism, in which a jog dislocation can create dislocation loops expanding on neighbouring planes and creating a twin embryo, has been proposed for FCC metals [11, 12, 13]. In BCC metals, the atomistic shear mechanism, which is the layer-by-layer accumulation of stacking faults, can take place [14]. In HCP metals a twin nucleus can be produced by the dissociation of a single dislocation on planes that are not parallel to the glide plane [15]. All these mechanisms require a resolved shear stress (RSS) proportional to $\gamma_{\mathrm{us}} / b$ where $\gamma_{\mathrm{us}}$ is the unstable stacking fault energy (maximum energy in the generalised stacking fault energy curve) and $b$ is the Burgers vector of the dissociated dislocations [16].

After nucleation, twin embryos grow and become thicker twin bands. This process involves the translation of the twin interface, which is aided by glissile dislocations called twinning partials [17]. The formation of a twinning partial may involve the interaction between a mobile dislocation and a twin interface [18], which is still poorly understood and not systematically studied [10]. Because of the Burgers vector conservation, this interaction leads to the formation of residual dislocations at the twin interface.

Twinning partials can also nucleate at the twin interface if the stress is sufficiently high [19]. The motion of twinning partials is responsible for twin propagation across a grain [18]. Twin growth is an incompletely understood mechanism. Specifically, the material parameters that determine twin growth have not been determined. Density functional theory (DFT) [14] and molecular dynamics (MD) simulations [18] are typically able to describe a twin with less than 10 atomic layers. Therefore, atomistic simulations of twin growth, as observed using electron microscopy, are rare in the literature because substantial computational resources would be required. The development of mesoscale models is therefore required.

An important observable is twin thickness, which can be measured using electron microscopy [20]. Some metals and alloys show few twins in each grain after deformation (e.g. Zirconium [21], Magnesium [22], Titanium [23]), with a characteristic thickness larger than $10 \mu \mathrm{m}$, while others show several thinner twins (e.g. Uranium [24], Copper-Aluminium [25], Copper-Germanium [26]) with a characteristic thickness ranging from a few nanometers to $5 \mu \mathrm{m}$. Multiple thin twins are also observed near grain boundaries [26, 20] and phase boundaries [27]. The average twin thickness 
increases with the grain size [28] and with the SFE in alloys [25].

Experimental evidences of twin nucleation and growth show that twins a few millimeters in thickness can form in less than a millisecond [29]. Transmission electron microscopy (TEM) observations show twins nucleating at phase boundaries from misfit dislocations [27]; their thickness is determined by the spacing between misfit dislocations along the boundary and by the dislocation-twin interaction energy. Twin growth in FCC metals due to the simultaneous emission of dislocations on a primary slip plane and a cross-slip plane has been observed during in-situ experiments [26].

Many questions still remain open: what is the difference between the stress necessary for twin nucleation and twin growth? How does the presence of a twin affect the nucleation of a neighbouring twin? Which material parameters determine twin thickness and spacing?

Mesoscale models have been developed that introduce possible physical mechanisms for twin nucleation and growth. Analytical models have been used to predict the twinning stress as a function of temperature, strain rate, grain size and SFE. Some models are based on the hypothesis that a dislocation pile-up is necessary to induce the twinning stress [30]. The number of twin bands in a grain as a function of the strain can be introduced as a variable and determined by minimisation of the Helmholtz free energy [31]. However, the description of the dynamic evolution of discrete twins requires simulations with a spatial resolution that is higher than the characteristic thickness of one twin band. Constitutive laws implemented in finite element solvers use the twin volume fraction as a state variable [32], which evolves based on the stress applied, therefore, the deformation due to twinning is averaged over continuum length scales. Discrete twins have been implemented by defining subsets of elements where twinning deformation occurs once a critical twin volume fraction or a critical macroscopic strain is reached [33, 34]. Remeshing algorithms have been used with this computational technique [35, 22]. These models are not able to predict the twin thickness because of the lack of an inherent physical length scale.

Phase field modelling, in which a variable $\varphi$ varies from 0 in the untwinned region to 1 in the twinned region, is suitable to describe the nucleation and growth of twins as no a priori assumptions on the location of the twin interface or remeshing are needed [36]. Phase field modelling has been used to describe single discrete twins using a stress relaxation effect, i.e. the critical resolved shear stress (CRSS) is reduced when $\varphi$ grows [37]. The coupling with grain growth models has been used to study thermal twins [38].

Mirkhani and Joshi implemented a specific slip law to describe the motion of twinning partials at the twin boundary interface in a continuum model [8]. This allows the growth of pre-existing twins to be reproduced. However, no internal length scales are introduced, therefore, only a single twin that grows indefinitely during deformation can be simulated, rather than a discrete pattern. The Helmholtz free energy has been introduced in phase field models to include the energy barrier for the formation of a twinning partial dislocation, the interfacial energy along the twin boundary and the interaction between twinning partials on different slip systems [39]. However, the role of mobile dislocations outside of the twin and the role of residual dislocations left at the twin interface were not investigated.

Phase field models can be coupled with the crystal plasticity finite element (CPFE) method $[40,41]$ and have been able to describe patterning phenomena related to dislocation dynamics $[42,43]$. A Ginzburg-Landau equation usually describes the time evolution of $\varphi$ [44]. The CPFE 
method includes plastic deformation due to slip, anisotropy of the elastic constants and a consistent elasto-plastic decomposition of the deformation [45].

In this paper, we develop a constitutive model able to describe discrete twin patterns emerging after deformation which is easily implemented in a CPFE framework [46]. A non-local term is introduced to calculate the stress for twin nucleation and growth at different spatial positions. The physical interpretation of this term is based on the interaction between twin interfaces with mobile and twinning dislocations. The dependence of the model parameters on the stacking fault energy (SFE) is also discussed. Because of this dependence, the model is not restricted to a single material. By changing the interaction parameters, we show that the simulated phase field $\varphi$ after deformation shows a transition between two states: one describing discrete twin patterning and the other describing a single large twin.

The paper is organised as follows: Section 2 describes the constitutive model for slip and twinning, Section 3 contains parametric studies and simulations of recent in-situ electron backscatter diffraction (EBSD) experiments [47]. Section 4 reports a discussion of the physical interpretation of the model for twin nucleation and growth. Specifically, the relationship between the model parameters and the stacking fault energy of different metals is discussed.

\section{Crystal plasticity finite element modelling}

A finite strain formulation is used in which the deformation gradient is decomposed into an elastic part and a plastic part describing a stress-free deformation given by slip and twinning [48]:

$$
\boldsymbol{F}=\mathbf{I}+\frac{\partial \mathbf{u}}{\partial \mathbf{X}}=\boldsymbol{F}_{e} \boldsymbol{F}_{p},
$$

where $\mathbf{u}=\boldsymbol{x}-\boldsymbol{X}$ is the displacement field relating a point in the initial, $\boldsymbol{X}$, and deformed, $\mathbf{x}$, material. The time evolution law of the plastic deformation gradient is written following the approach of Kalidindi [49]:

$$
\boldsymbol{L}_{p}=\dot{\boldsymbol{F}}_{p} \boldsymbol{F}_{p}^{-1}=\sum_{\alpha=1}^{N_{\text {slip }}} \dot{\gamma}_{\alpha}(\boldsymbol{\sigma}) \boldsymbol{s}_{\alpha} \otimes \boldsymbol{n}_{\alpha}+\dot{\varphi}(\boldsymbol{\sigma}) \gamma_{\beta}^{\mathrm{twin}} \boldsymbol{s}_{\beta} \otimes \boldsymbol{n}_{\beta} .
$$

$\boldsymbol{L}_{p}$ is known as the plastic velocity gradient [50]. $\boldsymbol{s}_{\alpha}$ and $\boldsymbol{n}_{\alpha}$ indicate the slip directions and the slip plane normals of the $N_{\text {slip }}$ slip systems. They are expressed in the intermediate configuration, i.e. after the application of $\boldsymbol{F}_{p}$ but before the application of $\boldsymbol{F}_{e}$. Similarly, the twinning slip direction is $\boldsymbol{s}_{\beta}$ and twin interface normal is $\boldsymbol{n}_{\beta}$, which has a maximum shear magnitude $\gamma_{\beta}^{\text {twin }}$. A single twin system will be used in the following simulations, but the model can be readily generalized to include additional twin systems by summing over $\beta$. $\dot{\gamma}_{\alpha}$ is the slip rate on the $\alpha$ slip system, while $\dot{\varphi}$ is the time evolution of the twin phase field, which is positive because detwinning is not allowed in the present model, even though it is observed in some materials [51]. The slip rate $\dot{\gamma}_{\alpha}$ in the first term of the right-hand side of Eq. (2) leads to plastic deformation both in the untwinned and in the twinned region. If slip inside the twinned regions is not considered, the stress would be slightly overestimated. The reorientation of the slip systems inside the twinned region is not taken into account because twinning is the dominant plastic deformation mechanism in those regions and because of computational efficiency. 


\subsection{Constitutive model for slip}

In this section the constitutive model to calculate the shear strain rate $\dot{\gamma}_{\alpha}(\sigma)$ and the CRSS for each slip system is reported. $\dot{\gamma}_{\alpha}(\sigma)$ follows a power-law relationship [52, 53]:

$$
\dot{\gamma}_{\alpha}(\sigma)=\dot{\gamma}_{0}\left|\frac{\tau_{\alpha}}{\tau_{\alpha}^{c}}\right|^{n} \operatorname{sign}\left(\tau_{\alpha}\right),
$$

where $\dot{\gamma}_{0}$ and $n$ are constants. They determine the strain rate dependence of the stress-strain response of the slip model. The simulations in the following will be carried out in a regime in which the model is weakly strain rate dependent. $\tau_{\alpha}=\boldsymbol{F}_{e}^{T} \boldsymbol{F}_{e} \mathbf{S}:\left(\boldsymbol{s}_{\alpha} \otimes \boldsymbol{n}_{\alpha}\right)$ is the RSS, calculated by contracting the tensor $\boldsymbol{F}_{e}^{T} \boldsymbol{F}_{e} \mathbf{S}$ on the Schmid tensor, where $\mathbf{S}$ is the second Piola-Kirchhoff stress, expressed in the intermediate configuration $[54,55]$. Details about the derivation of the resolved shear stress are reported in Appendix A. $\tau_{\alpha}^{c}$ is the CRSS of the slip system $\alpha . \dot{\gamma}_{\alpha}$ can be positive or negative depending on the term $\operatorname{sign}\left(\tau_{\alpha}\right)$.

A dislocation density based model is used to describe work hardening due to the slip system activity. The dislocation density evolution is based on the model originally developed by Beyerlein and Tomé for $\mathrm{Zr}$ [21], and the dislocation interactions are based on discrete dislocation dynamics simulations [56]. Two types of dislocation densities are used: $\rho_{\alpha}^{\text {for }}$, the forest dislocation density on slip system $\alpha$, and $\rho^{\text {sub }}$, the dislocation density in the substructure, such as dislocation cell walls [57]. The CRSS for the slip system $\alpha$ is given by [58, 59]:

$$
\tau_{\alpha}^{c}=\tau_{\alpha}^{0}+0.9 b_{\alpha} \mu_{\alpha} \sqrt{\rho_{\alpha}^{\text {for }}}+0.086 b_{\alpha} \mu_{\alpha} \sqrt{\rho^{\mathrm{sub}}} \log \left(\frac{1}{b_{\alpha} \sqrt{\rho^{\mathrm{sub}}}}\right),
$$

where $b_{\alpha}$ is the magnitude of the Burgers vector, $\mu_{\alpha}$ is the projected shear modulus of the slip system $\alpha[58]$ and $\tau_{\alpha}^{0}$ is a constant friction stress. The temperature dependence of $\tau_{\alpha}^{c}$ is not considered in the present model [60].

Work hardening is described by the following evolution laws for the forest and substructure dislocation densities [61]:

$$
\begin{aligned}
& \dot{\rho}_{\alpha}^{\text {for }}=k_{\alpha}^{1} \frac{\left|\dot{\gamma}_{\alpha}(\boldsymbol{\sigma})\right|}{b_{\alpha}}\left[\sqrt{\rho_{\alpha}^{\text {for }}}-\hat{d}_{\alpha} \rho_{\alpha}^{\text {for }}\right], \\
& \dot{\rho}^{\text {sub }}=1800 k_{1}^{1} \hat{d}_{1} \rho_{1}^{\text {for }} \sqrt{\rho^{\text {sub }}}\left|\dot{\gamma}_{1}(\sigma)\right|,
\end{aligned}
$$

where $k_{\alpha}^{1}$ is a pre-factor for dislocation multiplication and $\hat{d}_{\alpha}$ is a characteristic length scale for dislocation annihilation. The terms in Eqs. (5)-(6) represent dislocation multiplication and annihilation $[62,63]$. This model has been successfully used to reproduce the stress-strain curves of fine-grained $\alpha$-uranium [61]. Only the plastic shear on the first slip system (wall slip system) contributes to the growth of the dislocation density in the substructure. This is motivated by the fact that the wall slip system is the most active slip system in $\alpha$-uranium at room temperature. The prefactors $0.9,0.086,1800$ in Eqs. (4) and (6) are the same used by Tomé et al. for fine-grained $\alpha$-uranium [61]. Therefore, the dislocation multiplication parameters $k_{\alpha}^{1}$ used in (5) in the present work can be directly compared for different materials in the literature. 


\subsection{Constitutive model for twinning: free energy formulation}

The time evolution of the phase field $\dot{\varphi}$ in Eq. (2), needed to calculate the plastic deformation due to twinning, is found according to a thermodynamically consistent free energy formulation. Following the approach of Clayton, Knap [36] and Homayonifar, Mosler [31], the Helmholtz free energy can be decomposed into a term $\Psi_{\mathrm{e}}$, describing the elastic strain energy density, a term $\Psi_{\mathrm{pf}}$, describing the energy in twin bands and a term $\Psi_{\text {dis }}$, describing the stored energy associated with dislocations:

$$
\Psi=\Psi_{\mathrm{e}}\left(\mathbf{u}, \varphi, \rho_{\alpha}^{\mathrm{for}}, \rho^{\mathrm{sub}}\right)+\Psi_{\mathrm{pf}}\left(\varphi, \rho^{\text {total }}\right)+\Psi_{\mathrm{dis}}\left(\rho_{\alpha}^{\mathrm{for}}, \rho^{\mathrm{sub}}\right)
$$

$\Psi_{\text {dis }}$ is usually decomposed into self-hardening and latent hardening effects; it can be used to find the flow rule and hardening rate for plastic deformation due to slip [64]. In the present model we do not consider latent hardening of the slip systems due to twinning, therefore the term $\Psi_{\text {dis }}$ does not affect the time evolution of $\varphi$ and the variations $\delta_{\rho_{\alpha}^{\text {for }}} \Psi_{\mathrm{pf}}, \delta_{\rho^{\mathrm{sub}}} \Psi_{\mathrm{pf}}$ are considered negligible. The elastic strain energy density is given by the contraction of the second Piola-Kirchhoff stress and the elastic Green-Lagrange strain $\mathbf{E}_{e}[65]$ :

$$
\Psi_{\mathrm{e}}=\frac{1}{2} \mathbf{S}: \mathbf{E}_{e}=\frac{1}{2} \mathbf{E}_{e}: \mathbb{C}: \mathbf{E}_{e},
$$

where $\mathbb{C}$ is the stiffness tensor. Therefore, $\Psi_{\mathrm{e}}=\Psi_{\mathrm{e}}\left(\boldsymbol{F}_{e}\right)$ is a function of the elastic deformation gradient, $\boldsymbol{F}_{e}=\boldsymbol{F}_{e}\left(\mathbf{u}, \boldsymbol{F}_{p}\right)$, which is a function of the displacement field $\mathbf{u}$ and plastic deformation gradient $\boldsymbol{F}_{p} . \boldsymbol{F}_{p}=\boldsymbol{F}_{p}\left(\varphi, \rho_{\alpha}^{\text {for }}, \rho^{\text {sub }}\right)$ depends on the phase field $\varphi$, on $\rho_{\alpha}^{\text {for }}$ and on $\rho^{\text {sub }}$ because of the plastic deformation given by twinning and slip. Therefore, $\Psi_{\mathrm{e}}$ can be expressed as a function of $\mathbf{u}, \varphi, \rho_{\alpha}^{\text {for }}$ and $\rho^{\text {sub }}$, as in Eq. (7). The phase field contribution $\Psi_{\text {pf }}$ depends on the total dislocation density:

$$
\rho^{\text {total }}=\sum_{\alpha=1}^{N_{\text {slip }}} \rho_{\alpha}^{\text {for }}+\rho^{\text {sub }} .
$$

In the simulations in the present paper, a uniform initial dislocation density of $10^{10} \mathrm{~m}^{-2}$ was used. Additionally, an initial density $\rho_{0}(\boldsymbol{X})$ calculated from the crystal lattice misorientation [66], as measured by EBSD, was added to the initial value of the forest dislocation density $\rho_{1}^{\text {for }}$ on the first slip system (wall slip system):

$$
\rho_{\alpha}^{\text {for }}(t=0)= \begin{cases}10^{10} \mathrm{~m}^{-2}+\rho_{0}(X), & \text { if } \alpha=1, \\ 10^{10} \mathrm{~m}^{-2}, & \text { if } \alpha>1 .\end{cases}
$$

This choice is motivated by the fact that the wall slip system is the most active slip system in $\alpha$-uranium at room temperature. Assigning this dislocation density to another slip system would have a smaller effect on the slip behaviour. This choice does not affect the interaction between $\varphi$ and the total dislocation density $\rho^{\text {total }}$ described by $\Psi_{\text {pf }}$. The introduction of the initial density has been necessary to explain the experimental observations, as shown in Section 3.4.

The total dislocation density $\rho^{\text {total }}$ in Eq. (9) includes statistically stored dislocations (SSD) which evolve over time superimposed on a static GND density field measured experimentally [67] but this model does not explicitly calculate the evolution of GND density [68]. 
In the simulations in the present paper, the initial value of the dislocation density in the substructure is assumed to be uniform:

$$
\rho^{\mathrm{sub}}(t=0)=10^{10} \mathrm{~m}^{-2} .
$$

The stable configuration corresponds to the minimum of the free energy: the variation with respect to $\varphi$ is given by:

$$
\delta_{\varphi} \Psi=\delta_{\varphi} \Psi_{\mathrm{e}}\left(\mathbf{u}, \varphi, \rho_{\alpha}^{\mathrm{for}}, \rho^{\mathrm{sub}}\right)+\delta_{\varphi} \Psi_{\mathrm{pf}}\left(\varphi, \rho^{\text {total }}\right) .
$$

Detailed calculations of the partial derivatives of the elastic free energy $\Psi_{\mathrm{e}}$ with respect to the phase field are reported in Appendix B. This derivative is proportional to the RSS on the twin system $\tau_{\beta}$ :

$$
\delta_{\varphi} \Psi_{\mathrm{e}}=\frac{\partial \Psi_{\mathrm{e}}}{\partial \varphi} \delta \varphi=-\gamma_{\beta}^{\mathrm{twin}} \tau_{\beta} \delta \varphi .
$$

The free energy contribution $\Psi_{\mathrm{pf}}\left(\varphi, \rho^{\text {total }}\right)$ is decomposed into a term $\Psi_{\mathrm{pf}}^{0}(\varphi)$ describing twin nucleation and stress relaxation, a term $\Psi_{\text {twin }}(\varphi)$, representing the non-local term that describes twin growth, and a term $\Psi_{\text {twin-dis }}\left(\varphi, \rho^{\text {total }}\right)$ that describes the effect of the total dislocation density on twin nucleation:

$$
\Psi_{\mathrm{pf}}\left(\varphi, \rho^{\text {total }}\right)=\Psi_{\mathrm{pf}}^{0}(\varphi)+\Psi_{\text {twin }}(\varphi)+\Psi_{\text {twin-dis }}\left(\varphi, \rho^{\text {total }}\right) .
$$

The term $\Psi_{\text {twin }}(\varphi)$ describes the effect of a twin on the nucleation stress of additional twin layers at the twin interface. The term $\Psi_{\text {twin-dis }}\left(\varphi, \rho^{\text {total }}\right)$ describes the interaction between a twin and mobile dislocations, which leads to an additional stress term to nucleate and propagate a twin in the presence of mobile dislocations.

A quadratic form is chosen for the free energy contribution $\Psi_{\mathrm{pf}}^{0}(\varphi)$ :

$$
\Psi_{\mathrm{pf}}^{0}(\varphi)=\gamma_{\beta}^{\mathrm{twin}} \tau_{\beta}^{0} \begin{cases}\varphi-\frac{3}{4} \varphi^{2}, & \text { if } \varphi<\frac{1}{2}, \\ \frac{1}{4}+\frac{\varphi}{2}-\frac{3}{4} \varphi^{2}, & \text { if } \varphi>\frac{1}{2},\end{cases}
$$

where $\tau_{\beta}^{0}$ is a constant friction stress. Figure 1(a) shows the free energy $\Psi_{\mathrm{pf}}^{0}(\varphi)$ as a function of $\varphi$. The quadratic form for $\Psi_{\mathrm{pf}}^{0}(\varphi)$ is the simplest function that can describe an energy barrier that must be overcome to form a twin.

The physical meaning is the following: once the twin phase field has reached the value $1 / 2$, representing the maximum of the potential energy barrier that atoms have to overcome to reach their final equilibrium position in the twinned crystal [69], a driving force leads to complete twinning $(\varphi=1)$. The minimum free energy is therefore reached. For this reason, the last two terms in Eq. (14) $\Psi_{\text {twin }}$ and $\Psi_{\text {twin-dis }}$ contribute only if $\varphi<1 / 2$ and will be explained in detail in Sections 2.4 and 2.5 .

In a transformation in which the twin phase field grows at constant volume and temperature, the free energy decreases: $\delta_{\varphi} \Psi \leq 0$ [70]. Using Eqs. (13)-(15), when $\varphi<1 / 2$ :

$$
\left.\delta_{\varphi} \Psi\right|_{\varphi<1 / 2}=\gamma_{\beta}^{\mathrm{twin}}\left[-\tau_{\beta}+\tau_{\beta}^{c}\left(\varphi, \rho^{\mathrm{total}}\right)\right] \delta \varphi \leq 0 \Longrightarrow \delta \varphi=0 \text { or } \tau_{\beta} \geq \tau_{\beta}^{c}\left(\varphi, \rho^{\text {total }}\right) .
$$




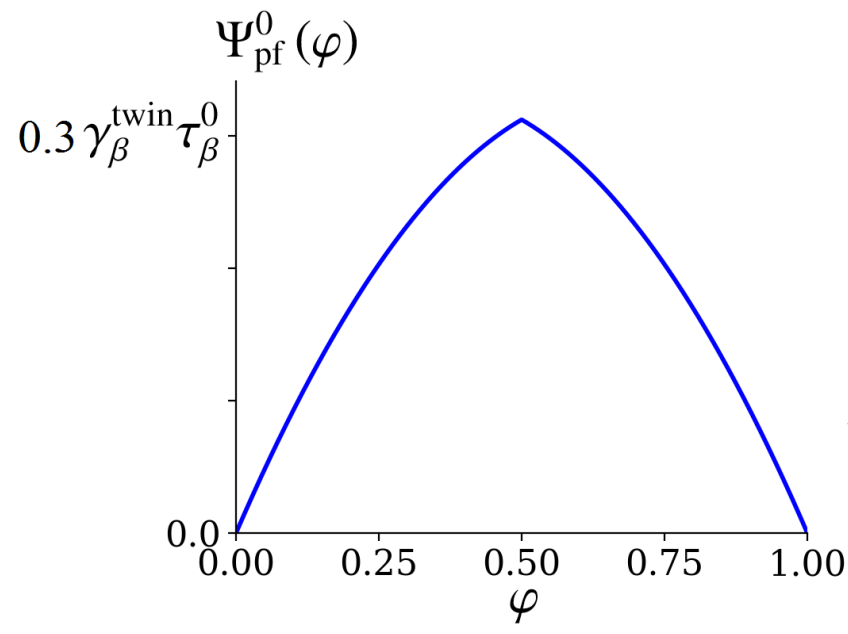

(a)

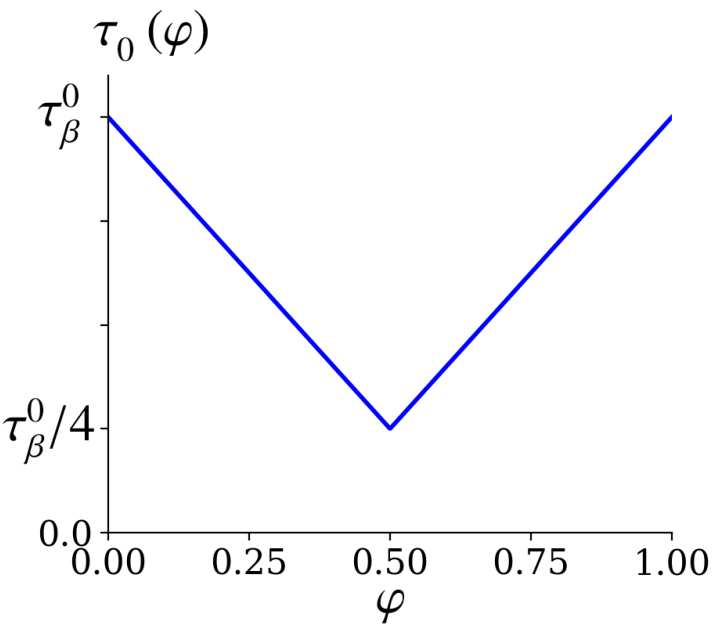

(b)

Figure 1: (a) Free energy term $\Psi_{\mathrm{pf}}^{0}$ of the twin phase field (Eq. 15). (b) The $\tau_{0}(\varphi)$ contribution to the CRSS (Eq. 18 and 19) for twinning due to twin nucleation and stress relaxation.

Therefore, the function $\tau_{\beta}^{c}\left(\varphi, \rho^{\text {total }}\right)$ gives the CRSS for twinning. The configuration is stable $(\dot{\varphi}=0)$ if the RSS on the twin system is less than $\tau_{\beta}^{c}\left(\varphi, \rho^{\text {total }}\right)$. The CRSS for twinning has three contributions:

$$
\tau_{\beta}^{c}\left(\varphi, \rho^{\text {total }}\right)=\frac{1}{\gamma_{\beta}^{\text {twin }}}\left(\frac{\partial \Psi_{\mathrm{pf}}^{0}}{\partial \varphi}+\frac{\partial \Psi_{\text {twin }}}{\partial \varphi}+\frac{\partial \Psi_{\text {twin-dis }}}{\partial \varphi}\right)=\tau_{0}(\varphi)+\tau_{\text {twin }}(\varphi)+\tau_{\text {twin-dis }}\left(\varphi, \rho^{\text {total }}\right),
$$

where:

$$
\left.\tau_{0}(\varphi)\right|_{\varphi<1 / 2}=\tau_{\beta}^{0}\left(1-\frac{3}{2} \varphi\right) .
$$

$$
\left.\frac{\partial \Psi}{\partial \varphi}\right|_{\varphi>1 / 2}=\gamma_{\beta}^{\mathrm{twin}}\left[-\tau_{\beta}+\tau_{\beta}^{0}\left(\frac{1}{2}-\frac{3}{2} \varphi\right)\right]<0 .
$$

The second term on the right hand side is always negative for $\varphi>1 / 2$, therefore complete twinning $(\varphi=1)$ can be reached even if no external stress is present.

The function $\tau_{0}(\varphi)$ can be extended to the interval $\varphi>1 / 2$ as shown in Figure 1 (b):

$$
\left.\tau_{0}(\varphi)\right|_{\varphi>1 / 2}=\tau_{\beta}^{0}\left(\frac{3}{2} \varphi-\frac{1}{2}\right) \text {. }
$$




\subsection{Constitutive model for twinning: rate equation}

Instead of using a Ginzburg-Landau equation for the time evolution of $\varphi$ towards the value that minimises the free energy, we use the following rate equation:

$$
\dot{\varphi}(\sigma, \varphi)=\dot{\varphi}_{S}(\sigma, \varphi)+\dot{\varphi}_{G}(\varphi) .
$$

The first term is stress-driven and it is given by the power law:

$$
\gamma_{\beta}^{\mathrm{twin}} \dot{\varphi}_{S}(\sigma, \varphi)= \begin{cases}\dot{\gamma}_{0}\left|\frac{\tau_{\beta}}{\tau_{\beta}^{c}}\right|^{n}, & \text { if } \tau_{\beta}>0 \\ 0, & \text { if } \tau_{\beta}<0\end{cases}
$$

A large value of the exponent $n$ in the power law ensures that the condition $\delta \varphi>0 \Longleftrightarrow \tau_{\beta}>\tau_{\beta}^{c}$ in Eq. (16) is satisfied. This term becomes relevant when the RSS $\tau_{\beta}$ on the twin system is higher than the critical value $\tau_{\beta}^{c}$.

The second term is given by:

$$
\dot{\varphi}_{G}(\varphi)= \begin{cases}f(1-\varphi), & \text { if } \varphi>\frac{1}{2}, \\ 0, & \text { if } \varphi<\frac{1}{2},\end{cases}
$$

where $1 / f$ represents the characteristic time at which the twinning process completes after the twin phase field has reached the value 1/2, as explained in Section 2.2. This characteristic time is independent of the applied stress. This second term describes the time evolution of the twin phase field in the interval $1 / 2<\varphi<1$ and represents the force that drives the system towards the minimum free energy $\Psi_{\mathrm{pf}}^{0}(\varphi)$ when $\varphi>1 / 2$ in Figure 1 (a). Therefore, the increase in $\tau_{0}(\varphi)$ in the interval $1 / 2<\varphi<1$ does not restrict the formation of a complete twin. The characteristic time $1 / f$ chosen is much smaller than the total simulation time but larger than the maximum time increment $(0.04 \mathrm{~s})$. Thus, the twinning process can be integrated properly over time in the present simulations, even if the time increment used is longer than the experimental propagation time [72].

The difference between this approach and the Ginzburg-Landau rate equation for the twin phase field [40], in which $\dot{\varphi}$ is directly proportional to $\partial \Psi_{\mathrm{e}} / \partial \varphi \propto \tau_{\beta}$, is described in the following. The power law relationship (22) guarantees that twinning will take place when the RSS is close to the CRSS and eliminates the rate sensitivity of the model. If a Ginzburg-Landau approach is used [40], the time evolution of $\varphi$ is directly proportional to the RSS, leading to a critical stress for twinning that is directly proportional to the strain rate. This strong strain rate dependence is not observed experimentally [73, 74].

Both Eq. (21) and the Ginzburg-Landau rate equation satisfy the thermodynamics condition in Eq. (16), that is, the time evolution of the twin phase field leads to a decrease of the free energy.

\subsection{Non-local term to describe twin growth}

A non-local term is introduced to describe the effect of a twin on the nucleation stress for a neighbouring twin:

$$
\tau_{\text {twin }}(\varphi)=\frac{\tau_{\text {twin }}^{0}}{\Omega}\left(\int_{\Omega} \varphi d V\right),
$$


2.5. Interaction between twins and local dislocation density

The local interaction between a twin and dislocations, which is the additional stress to nucleate a twin in the presence of the local stress field of dislocations, $\tau_{\text {twin-dis }}\left(\varphi, \rho^{\text {total }}\right)$, depends linearly

where the integral is calculated over a cylindrical neighbourhood of the point of interest, as shown in Figure $2(\mathrm{~b}), \Omega$ is the volume of the cylinder and $\tau_{\text {twin }}^{0}$ is a constant. Only integration points where the atoms have overcome the potential energy barrier $\left(\varphi>\frac{1}{2}\right)$ are considered when evaluating the integral. The axis of the cylindrical volume $\Omega$ is chosen parallel to the twin plane normal. Thus, the integral in Eq. (24) is proportional to the sum of the thickness of the twins in the neighbourhood of the point of interest.

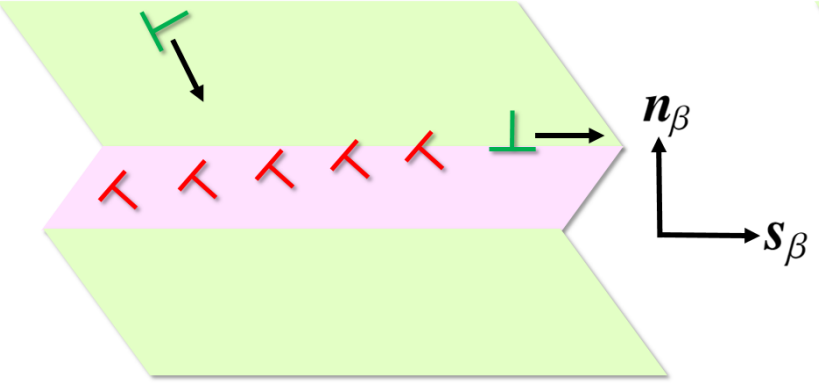

(a)

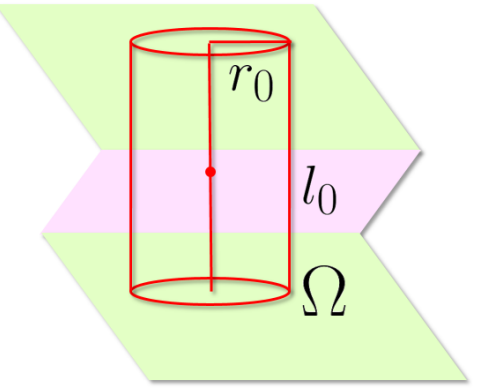

(b)
Figure 2: (a) Dislocation-based mechanism for twin growth. (b) Cylindrical integration volume used to calculate the CRSS for twinning.

The non-local term in (24) is used to model the following process, shown in Figure 2 (a): a mobile dislocation interacts with a twin interface, forming a mobile twinning dislocation and an immobile residual dislocation [18]. The motion of the twinning dislocation forms a new twin layer. The residual dislocation remains connected to the moving twinning dislocation through a stacking fault and the stress necessary to move the twinning dislocation is modelled by the term $\tau_{0}(\varphi)$ in Eq. (17). Residual dislocations produce a stress field that can prevent further mobile dislocations from reaching the twin interface. Therefore, this stress field has to be added to the twinning CRSS and it is represented by $\tau_{\text {twin }}(\varphi)$ in Eq. (17). Each residual dislocation is responsible for the creation of an additional twin layer. The mechanism described implies that the number of residual dislocations is directly proportional to the twin thickness, therefore the stress term $\tau_{\text {twin }}(\varphi)$ should be also proportional to the twin thickness. This is obtained by assuming a linear relationship between $\tau_{\text {twin }}(\varphi)$ and the integral of the phase field $\varphi$ over the cylindrical volume, which is proportional to the sum of the thickness of the twins in the neighbourhood of the point of interest. The length $l_{0}$ represents the characteristic length scale of the stress field induced by the presence of dislocations [75]. The calibration of the parameter $\tau_{\text {twin }}^{0}$ is obtained by comparison with in-situ EBSD experiments, as explained in Section 3.3. Other types of dislocations inside the twin, different from the residual dislocations, may also contribute to the stress term $\tau_{\text {twin }}(\varphi)$. In the present work, we want to show that including the residual dislocations is sufficient to model discrete twins, as observed in the EBSD experiment. 
on the total dislocation density $\rho^{\text {total }}$, as previously determined using digital image correlation experiments [74]:

$$
\tau_{\text {twin-dis }}\left(\varphi, \rho^{\text {total }}\right)=k_{\beta} \mu_{\beta} b_{\beta} b_{\alpha} \rho^{\text {total }},
$$

\subsection{Stiffness tensor and numerical implementation}

An implicit finite element implementation of the CPFE method, written as a UMAT for Abaqus, is used [46]. The equilibrium equation for the Cauchy stress $\sigma$ is solved:

$$
\nabla \cdot \sigma=\mathbf{0} .
$$

At every time increment $\Delta t$, the stress increment is calculated using the Jaumann stress rate and it is given by:

$$
\begin{aligned}
\Delta \boldsymbol{\sigma} & =\mathbb{C} \Delta \boldsymbol{\varepsilon}_{e l}+\left(\boldsymbol{W}_{e} \boldsymbol{\sigma}_{0}-\boldsymbol{\sigma}_{0} \boldsymbol{W}_{e}\right) \Delta t \\
& =\mathbb{C}\left(\Delta \boldsymbol{\varepsilon}-\Delta \boldsymbol{\varepsilon}_{p l}(\boldsymbol{\sigma})\right)+\left(\boldsymbol{W}_{e} \boldsymbol{\sigma}_{0}-\boldsymbol{\sigma}_{0} \boldsymbol{W}_{e}\right) \Delta t,
\end{aligned}
$$

where $\sigma_{0}$ is the Cauchy stress at the previous time increment and $\boldsymbol{W}_{e}$ is the elastic continuum spin $[77,58] . \mathbb{C}$ is the stiffness tensor, whose components are reported in Tab. 2 in Voigt notation. $\Delta \varepsilon$, $\Delta \boldsymbol{\varepsilon}_{e l}$ and $\Delta \boldsymbol{\varepsilon}_{p l}$ are the total, elastic and plastic parts of the small strain increment respectively. They are found by calculating the symmetric part of the total and plastic velocity gradients:

$$
\begin{gathered}
\Delta \boldsymbol{\varepsilon}=\frac{1}{2}\left(\boldsymbol{L}+\boldsymbol{L}^{T}\right) \Delta t \\
\Delta \boldsymbol{\varepsilon}_{p l}(\sigma)=\frac{1}{2}\left(\boldsymbol{L}_{p}(\boldsymbol{\sigma})+\boldsymbol{L}_{p}^{T}(\boldsymbol{\sigma})\right) \Delta t .
\end{gathered}
$$

$\boldsymbol{L}_{p}(\boldsymbol{\sigma})$ is calculated as in Eq. (2) while the total velocity gradient $\boldsymbol{L}$ is given by:

$$
\boldsymbol{L}=\dot{\boldsymbol{F}} \boldsymbol{F}^{-1} \text {. }
$$

Eq. (27) is an implicit equation for the stress increment $\Delta \sigma$ that is solved iteratively by a NewtonRaphson algorithm [46]. Specifically, the algorithm has to find the zero of the entries of the [3 $\times 3$ ] residual matrix $\Phi(\Delta \sigma)$ :

$$
\begin{aligned}
\mathbf{0}=\boldsymbol{\Phi}(\Delta \boldsymbol{\sigma})= & \mathbb{C}\left(\Delta \boldsymbol{\varepsilon}-\Delta \boldsymbol{\varepsilon}_{p l}(\boldsymbol{\sigma})\right) \\
& +\left(\boldsymbol{W}_{e} \boldsymbol{\sigma}_{0}-\boldsymbol{\sigma}_{0} \boldsymbol{W}_{e}\right) \Delta t-\Delta \boldsymbol{\sigma}
\end{aligned}
$$


For small elastic strain, the increment of the elastic part of the small strain $\Delta \boldsymbol{\varepsilon}_{e l}$ is approximately the same as the increment of the elastic Green-Lagrange strain $\mathbf{E}_{e}$. In the present simulations, the elastic strain is always smaller than about $0.05 \%$. Therefore, the stress expressed by Eq. (8) and the one calculated using the Jaumann stress rate are approximately the same [46].

The Jacobian used in the Newton-Raphson algorithm is given by [46, 58]:

$$
\frac{\partial \boldsymbol{\Phi}(\Delta \sigma)}{\partial \Delta \boldsymbol{\sigma}}=-\mathbb{C} \frac{\partial \Delta \boldsymbol{\varepsilon}_{p l}(\boldsymbol{\sigma})}{\partial \boldsymbol{\sigma}}-\mathbf{I} .
$$

The difference between this numerical implementation and the one described in [58], in which twinning was described as a homogeneous field, lies in the choice of the initial stress increment. In the current model, the stress increment $\Delta \sigma$ in the first iteration of the Newton-Raphson algorithm is assumed to be zero and not completely elastic. This has been necessary to obtain convergence using the constitutive model described by Eq. (18), in which the twinning CRSS decreases with increasing phase field $\varphi$ and stress relaxation takes place.

The crystal lattice reorientation due to twinning is taken into account, whereby the stiffness tensor used in Eq. (27) is a linear function of the twin phase field $\varphi$ :

$$
\mathbb{C}=(1-\varphi) \mathbb{C}_{\text {mat }}+\varphi \mathbb{C}_{\text {twin }},
$$

where $\mathbb{C}_{\text {mat }}$ and $\mathbb{C}_{\text {twin }}$ are the stiffness tensors in the original lattice (matrix) and twinned lattice respectively. They are related by the lattice rotation represented by the matrix [78]:

$$
\begin{gathered}
\mathbf{Q}=2 \boldsymbol{n}_{\beta} \otimes \boldsymbol{n}_{\beta}-\mathbf{I}, \\
{\left[\mathbb{C}_{\mathrm{twin}}\right]_{i j k l}=[\mathbf{Q}]_{i m}[\mathbf{Q}]_{j n}[\mathbf{Q}]_{k p}[\mathbf{Q}]_{l q}\left[\mathbb{C}_{\text {mat }}\right]_{m n p q},}
\end{gathered}
$$

where summation over repeated indices is assumed.

\subsection{Material parameters}

$\alpha$-uranium (orthorhombic) material parameters are used in the following simulations [58] because the model has been calibrated using experimental investigations on this material, as explained in Section 3.3. The slip systems and twin system used in the simulations are reported in Table 1.

The elastic constants $\mathbb{C}_{\text {mat }}$ are reported in Table 2 while the other model parameters are in Table 3.

\section{Simulation details and results}

The following simulations are made with a maximum time increment of $0.04 \mathrm{~s}$ and the strain rate, for both the shear and tensile simulations, is $10^{-3} \mathrm{~s}^{-1}$. In this regime the model is strain rate independent. A random generator is used to assign the initial twin phase field $\varphi$ in an interval between 0 and 0.001 to all integration points. All the simulations in the following are 3D with a single element across the thickness. 


\begin{tabular}{|l|c|c|}
\hline Slip system & $\boldsymbol{s}_{\alpha}^{0}$ & $\boldsymbol{n}_{\alpha}^{0}$ \\
\hline$\alpha=1$ (wall) & {$[1,0,0]$} & {$[0,1,0]$} \\
$\alpha=2$ (floor) & {$[1,0,0]$} & {$[0,0,1]$} \\
$\alpha=3$ (chimney) & {$[0.437,-0.899,0]$} & {$[0.899,0.437,0]$} \\
$\alpha=4$ (chimney) & {$[0.437,0.899,0]$} & {$[0.899,-0.437,0]$} \\
$\alpha=5$ (roof) & {$[0.241,-0.495,0.835]$} & {$[0.0,0.860,0.510]$} \\
$\alpha=6$ (roof) & {$[-0.241,-0.495,0.835]$} & {$[0.0,0.860,0.510]$} \\
$\alpha=7$ (roof) & {$[0.241,0.495,0.835]$} & {$[0.0,0.860,-0.510]$} \\
$\alpha=8$ (roof) & {$[0.241,-0.495,-0.835]$} & {$[0.0,0.860,-0.510]$} \\
\hline Twin system & $\boldsymbol{s}_{\beta}^{0}$ & $\boldsymbol{n}_{\beta}^{0}$ \\
\hline$\beta=1$ & {$[0.825,-0.565,0]$} & {$[0.565,0.825,0]$} \\
\hline
\end{tabular}

Table 1: Slip and twin systems used in the model [61]. The directions and plane normals are expressed in Cartesian coordinates and in the lattice coordinate system.

\begin{tabular}{|c|c|c|c|c|c|c|c|c|}
\hline $\mathbb{C}_{11}$ & $\mathbb{C}_{12}$ & $\mathbb{C}_{13}$ & $\mathbb{C}_{22}$ & $\mathbb{C}_{23}$ & $\mathbb{C}_{33}$ & $\mathbb{C}_{44}$ & $\mathbb{C}_{55}$ & $\mathbb{C}_{66}$ \\
\hline 214.74 & 46.49 & 21.77 & 198.57 & 107.91 & 267.11 & 124.44 & 73.42 & 74.33 \\
\hline
\end{tabular}

Table 2: Elastic constants (GPa) at room temperature for the orthorhombic structure of $\alpha$-uranium [79, 80] in Voigt notation (untwinned crystal lattice).

\subsection{Dependence on $l_{0}$}

Pure shear single crystal simulations are carried out to find the dependence on the model parameter $l_{0}$. The values $l_{0}=10,20,30 \mu \mathrm{m}$ are used. A single twin system is assumed: the twin direction $s_{\beta}$ is aligned with the $\mathrm{x}$ axis, while the twin plane normal is aligned with the $\mathrm{y}$ axis in figure 3. The parameter $\tau_{\text {twin }}^{0}=2000 \mathrm{MPa}$ has been used in these simulations.

The boundary conditions used are the following: $u_{x}=u_{y}=0$ on the surface $y=0, u_{z}=0$ on the surface $z=0$ and $u_{x}$ increasing from 0 to $30 \mu \mathrm{m}$ on the surface $y=100 \mu \mathrm{m}$.

The twin phase field at 30\% shear strain is shown in Figure 3. As shown in Figure 3, a twin pattern appears with approximately equispaced twins (red regions where $\varphi=1$ ): the larger the parameter $l_{0}$, the larger the spacing between neighbouring twins. Moreover, twins become thicker when $l_{0}$ is increased and the total twin volume fraction at $30 \%$ strain is approximately the same in all three cases.

This shows that the non-local term in Eq. (24) can stop the growth of a twin in a direction parallel to the twin plane normal.

\subsection{Dependence on the maximum twin-twin interaction stress $\tau_{\text {twin }}^{0}$}

Pure tension single crystal simulations are carried out to find the dependence of the model on the parameter $\tau_{\text {twin }}^{0}$. The values $\tau_{\text {twin }}^{0}=50,600,2000 \mathrm{MPa}$ are used. The boundary conditions and crystal orientation are motivated by recent in-situ EBSD experiments [47].

The size of the representative volume is $120 \mu \mathrm{m} \times 120 \mu \mathrm{m} \times 1 \mu \mathrm{m}$. The boundary conditions used are the following: $u_{x}=0$ on the surface $x=0, u_{y}=u_{z}=0$ at the point $(0,0,0), u_{y}=0$ at the point $(0,0,1 \mu \mathrm{m}), u_{z}=0$ at the point $(0,120 \mu \mathrm{m}, 0)$ and $u_{x}$ increasing from 0 to $24 \mu \mathrm{m}$ on the surface $x=120 \mu \mathrm{m}$. 


\begin{tabular}{|c|c|}
\hline Slip law parameters & Eq. (3) \\
\hline Plastic strain rate coefficient $\left(\dot{\gamma}_{0}\right)$ & $0.001 \mathrm{~s}^{-1}$ \\
\hline Plastic strain rate exponent $(n)[67]$ & 20 \\
\hline Twin law parameters & Eqs. (18),(19),(22),(23) \\
\hline Constant friction stress (twinning) $\left(\tau_{\beta}^{0}\right)$ & $25 \mathrm{MPa}$ \\
\hline Magnitude of shear due to twinning $\left(\gamma_{\beta}^{\mathrm{twin}}\right)[24]$ & 0.299 \\
\hline Characteristic time $(1 / f)$ & $1 \mathrm{~s}$ \\
\hline Slip hardening law parameters & Eq. (4) \\
\hline Constant friction stress (wall slip) $\left(\tau_{1}^{0}\right)[74]$ & $24.5 \mathrm{MPa}$ \\
\hline Constant friction stress (floor slip) $\left(\tau_{2}^{0}\right)[74]$ & $85.5 \mathrm{MPa}$ \\
\hline Constant friction stress (chimney slip) $\left(\tau_{3}^{0} ; \tau_{4}^{0}\right)[74]$ & $166.5 \mathrm{MPa}$ \\
\hline Constant friction stress (roof slip) $\left(\tau_{5}^{0} ; \tau_{6}^{0} ; \tau_{7}^{0} ; \tau_{8}^{0}\right)[81]$ & $235 \mathrm{MPa}$ \\
\hline Burgers vector (wall slip) $\left(b_{1}\right)[82]$ & $0.285 \mathrm{~nm}$ \\
\hline Burgers vector (floor slip) $\left(b_{2}\right)[82]$ & $0.285 \mathrm{~nm}$ \\
\hline Burgers vector (chimney slip) $\left(b_{3} ; b_{4}\right)[82]$ & $0.651 \mathrm{~nm}$ \\
\hline Burgers vector (roof slip) $\left(b_{5} ; b_{6} ; b_{7} ; b_{8}\right)[82]$ & $1.185 \mathrm{~nm}$ \\
\hline Projected shear modulus (wall slip) $\left(\mu_{1}\right)[79]$ & $74.330 \mathrm{GPa}$ \\
\hline Projected shear modulus (floor slip) $\left(\mu_{2}\right)[79]$ & $73.420 \mathrm{GPa}$ \\
\hline Projected shear modulus (chimney slip) $\left(\mu_{3}, \mu_{4}\right)[79]$ & $92.255 \mathrm{GPa}$ \\
\hline Projected shear modulus (roof slip) $\left(\mu_{5}, \mu_{6}, \mu_{7}, \mu_{8}\right)[79]$ & $115.67 \mathrm{GPa}$ \\
\hline Dislocation density evolution law parameters & Eqs. (5),(6) \\
\hline Dislocation multiplication prefactor (wall slip) $\left(k_{1}^{1}\right)[74]$ & 0.0121 \\
\hline Dislocation multiplication prefactor (floor slip) $\left(k_{2}^{1}\right)[74]$ & 0.36 \\
\hline Dislocation multiplication prefactor (chimney slip) $\left(k_{3}^{1} ; k_{4}^{1}\right)[74]$ & 0.136 \\
\hline Dislocation multiplication prefactor (roof slip) $\left(k_{5}^{1} ; k_{6}^{1} ; k_{7}^{1} ; k_{8}^{1}\right)[61]$ & 0.948 \\
\hline Dislocation annihilation length (wall slip) $\left(\hat{d}_{1}\right)[61]$ & $0.936 \mu \mathrm{m}$ \\
\hline Dislocation annihilation length (floor slip) $\left(\hat{d}_{2}\right)[61]$ & $0.429 \mu \mathrm{m}$ \\
\hline Dislocation annihilation length (chimney slip) $\left(\hat{d}_{3}, \hat{d}_{4}\right)[61]$ & $0.174 \mu \mathrm{m}$ \\
\hline Dislocation annihilation length (roof slip) $\left(\hat{d}_{5}, \hat{d}_{6}, \hat{d}_{7}, \hat{d}_{8}\right)[61]$ & $0.124 \mu \mathrm{m}$ \\
\hline Non-local term to describe twin growth & Eq. (24) \\
\hline Radius of the cylindrical neighbourhood $\left(r_{0}\right)$ & $1 \mu \mathrm{m}$ \\
\hline Length of the cylindrical neighbourhood $\left(l_{0}\right)$ & $10 \mu \mathrm{m}$ \\
\hline Maximum twin-twin interaction stress $\left(\tau_{\mathrm{twin}}^{0}\right)$ & sec. 3.2 \\
\hline Twin-dislocations local interaction law & Eq. (25) \\
\hline Twin Burgers vector $\left(b_{\beta}\right)$ & $0.1036 \mathrm{~nm}$ \\
\hline $\begin{array}{l}\text { Twin system projected shear modulus }\left(\mu_{\beta}\right) \\
\text { Dislocation-twin interaction coefficient } k_{\beta}\end{array}$ & $\begin{array}{l}99.537 \mathrm{GPa} \\
\text { sec. } 3.4\end{array}$ \\
\hline
\end{tabular}

Table 3: Model parameters. 


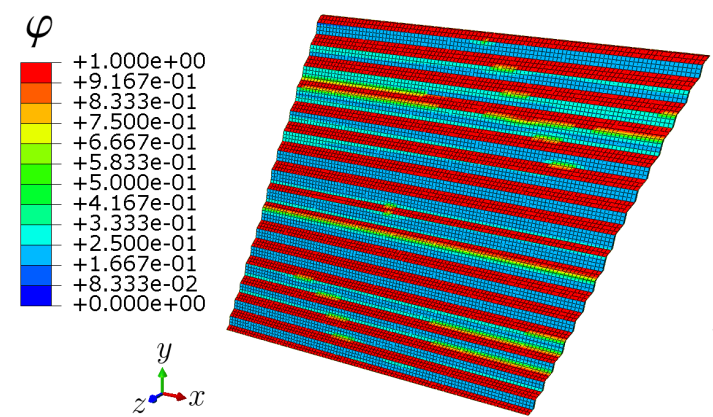

(a) $l_{0}=10 \mu \mathrm{m}$

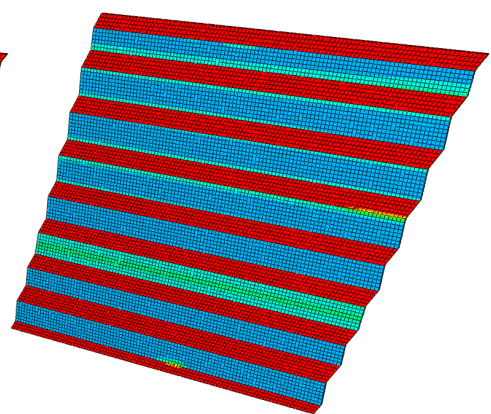

(b) $l_{0}=20 \mu \mathrm{m}$

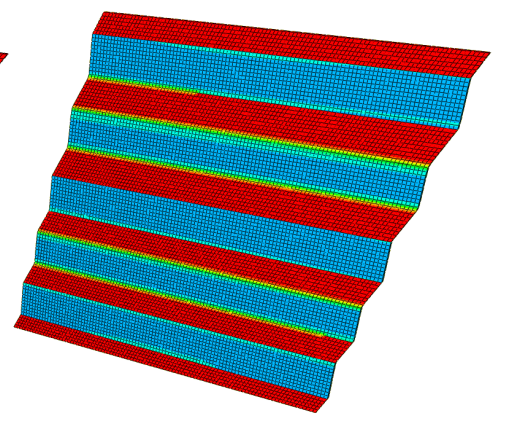

(c) $l_{0}=30 \mu \mathrm{m}$

Figure 3: Twin phase field under simple shear at $30 \%$ strain. Deformation is magnified by a factor 2 . The height of the simulated volume is $100 \mu \mathrm{m}$.

A single twin system, as reported in Table 1, is used and the crystal rotation matrix, which transforms a vector in the lattice reference frame to a vector in the sample reference frame $(x, y, z)$, is:

$$
R=\left(\begin{array}{ccc}
-0.6609 & -0.7454 & 0.0868 \\
-0.1228 & -0.0067 & -0.9924 \\
0.7403 & -0.6666 & -0.0872
\end{array}\right)
$$

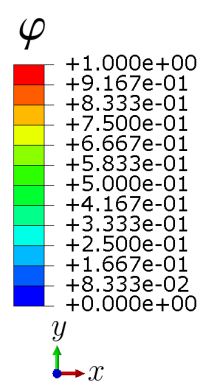

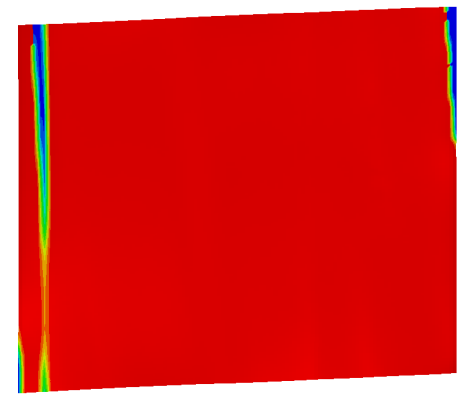

(a) $\tau_{\text {twin }}^{0}=50 \mathrm{MPa}$

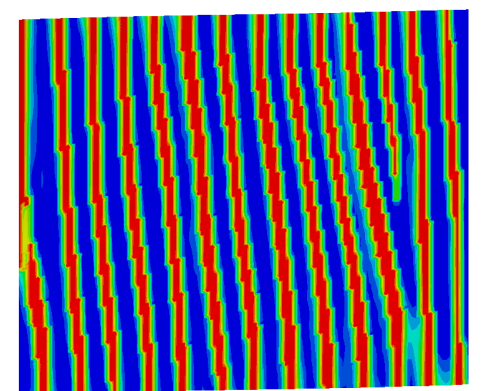

(b) $\tau_{\text {twin }}^{0}=600 \mathrm{MPa}$

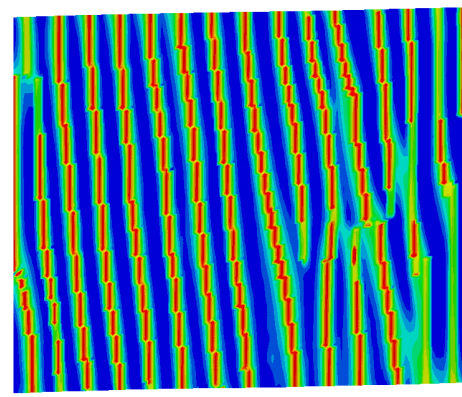

(c) $\tau_{\text {twin }}^{0}=2000 \mathrm{MPa}$

Figure 4: Twin phase field after $20 \%$ tensile strain along $x$. Dependence on the parameter $\tau_{\text {twin }}^{0}$.

$\varphi$ no longer forms a discrete pattern. Instead, a single large twin develops occupying nearly the entire simulated volume. The asymmetry in the twin development in Fig. 4 (b)-(c) is due to the introduction of the initial total dislocation density, as will be explained in more detail in Section 3.4. The single large twin in Fig. 4 (a) is given by several discrete twins combining into a single one. By contrast, in Fig. 4 (c), twins do not merge because the stress required would be too high. 


\subsection{Simulation of the in-situ mechanical test}

In-situ EBSD experiments have been carried out on a $\alpha$-uranium dogbone sample [47], as shown in Fig. 5 (a). EBSD scans, with a resolution of $0.5 \mu \mathrm{m}$, are taken from a region of interest with size $120 \mu \mathrm{m} \times 120 \mu \mathrm{m}$ at different applied strain. An estimation of the GND density in the undeformed sample is shown in Fig. 5 (b) [66]. This density is directly proportional to the kernel average misorientation, which is the average misorientation around a measurement point with respect to a defined set of nearest neighbour points [83]. Calculations have been carried out using the MTEX package [84].

The blue spots (region 2), where the initial GND density is set to zero, correspond to nonindexed regions of the EBSD map, which may be caused by the presence of oxide particles. A low angle boundary (vertical feature in region 1) is observed on the left part and it is modelled as a large concentration of initial GND. Because the state variables in the present model do not distinguish between GND and SSD, this initial density is added as forest dislocations on the wall slip system $\left(\rho_{0}(\boldsymbol{X})\right)$, as explained in Section 2.2. The EBSD experiment can access only the GND density and not the total dislocation density; therefore, the measured initial GND density has to be taken as a lower limit for the total dislocation density.

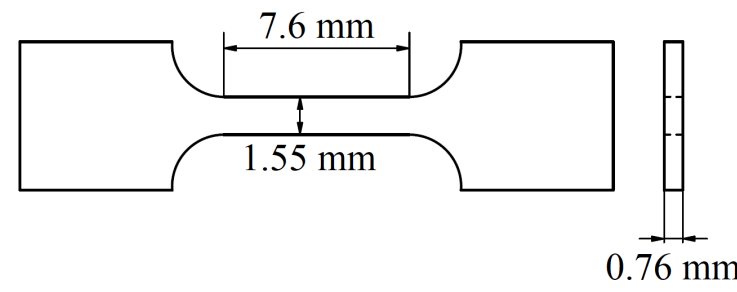

(a)

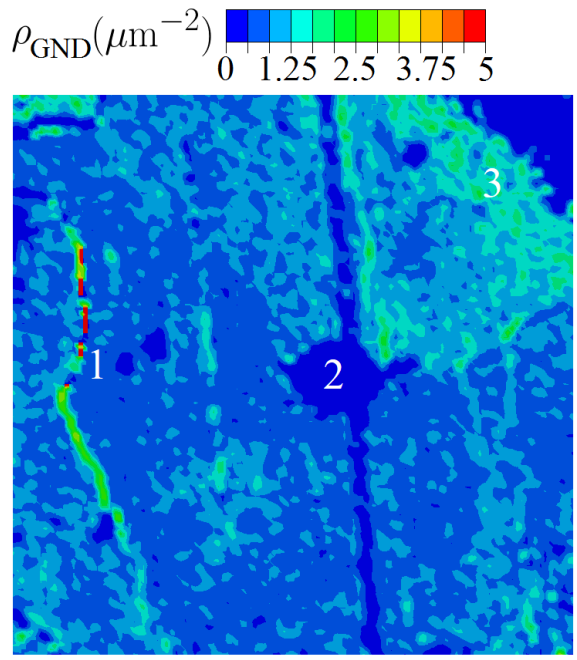

$50 \mu \mathrm{m}$

(b)

Figure 5: (a) Sample drawing. (b) Initial density of geometrically necessary dislocations (GND) in the region of interest: 1) low angle boundary; 2) non-indexed region; 3) high GND density region.

The measured average crystal orientation is represented by the rotation matrix $R$ in Eq. (36) and this has been used for the simulations. The mesh used has element size $0.5 \mu \mathrm{m}$, corresponding to the resolution of the EBSD scans. Pure tension simulations, which reproduce the experimental boundary conditions, are carried out to find the time evolution of the twin phase field. The boundary conditions used are the same described in section 3.2. The comparison between the experimentally observed twin pattern and the simulated twin phase field is shown in Fig. 6. 


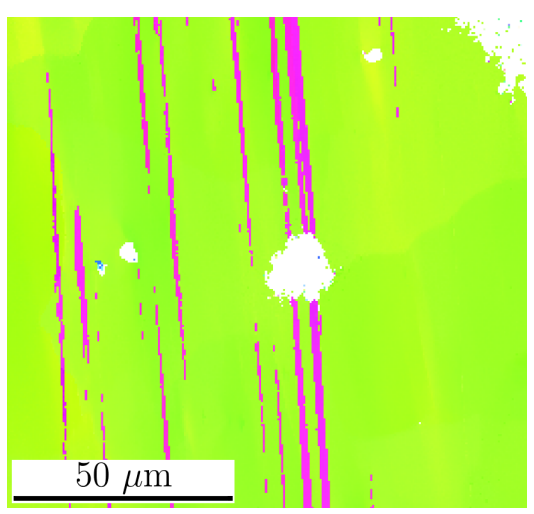

(a)

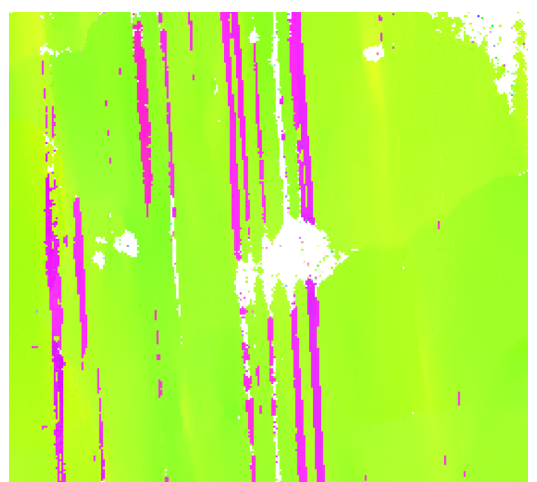

(c)

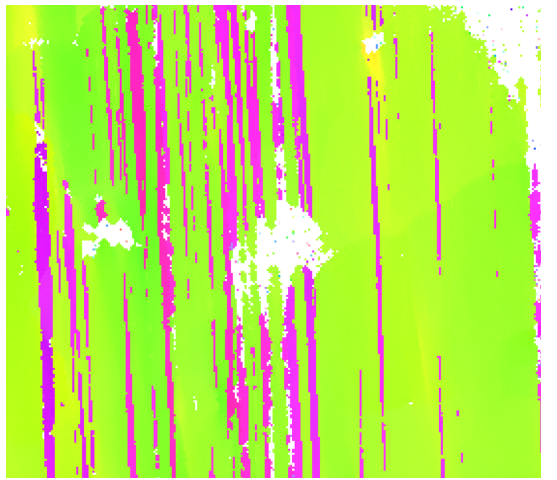

(e)

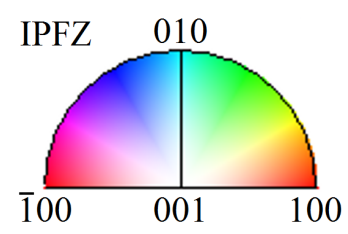

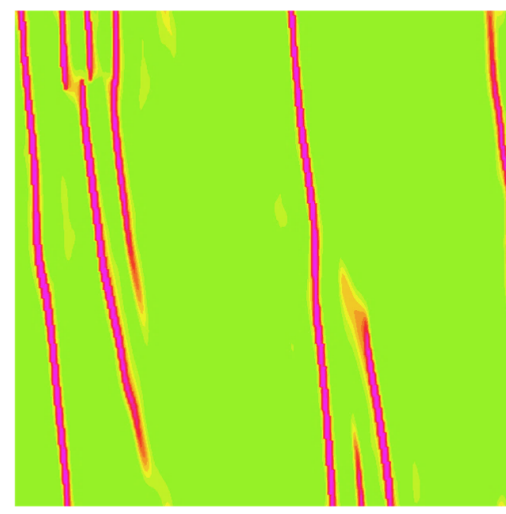

(b)

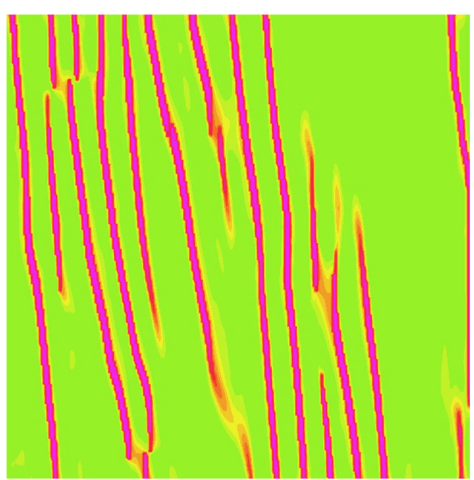

(d)

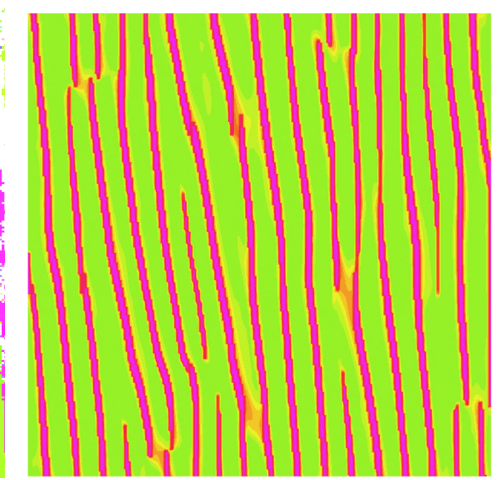

(f)

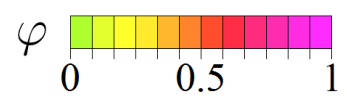

Figure 6: Comparison between experimental and simulated twin pattern at strain (a)-(b) 7\%, (c)-(d) 9\%, (e)-(f) $14 \%$. 
The colors in the experimental images in the left column of Fig. 6 correspond to the colormap of the inverse pole figure. In the images in the right column of Fig. 6, the colormap of the simulated twin phase field has been adjusted to produce light green for $\varphi=0$ and purple for $\varphi=1$. This choice is made for easier comparison between simulations and experiments.

The following features can be seen in both experiment and simulation in Fig. 6. Twins nucleate in the centre of the volume, near the circular non-indexed region 2. More twins are present in the left part of the geometry at $7 \%$ strain. This is due to the local twin-dislocations interaction term in Eq. (25). Indeed, the initial GND density has higher values on the top-right part (region 3) of the representative volume and along the low angle boundary (region 1), as shown in Fig. 5 (b). These are the regions in which the initial twin nucleation is prevented. At $9 \%$ strain, simulated twins have also nucleated in the vicinity of the low angle boundary (region 1) and are still prevalent in the left part of the geometry, as shown in Fig. 6 (d). At 14\% strain, twins have nucleated everywhere in the region of interest and initial twins have become thicker.

As shown in Section 3.1, the choice of the parameter $l_{0}$ is important to reproduce the number of twins after deformation. Similarly, as shown in Section 3.2, the choice of $\tau_{\text {twin }}^{0}$ determines the twin thickness.

The number of twins as a function of the applied strain is shown in Fig. 7. The number of twins is counted along horizontal lines in Fig. 6 and then averaged. The uncertainty in the experimental data is mainly due to the uncertainty on the local strain. The choice $l_{0}=10 \mu \mathrm{m}$ and $\tau_{\text {twin }}^{0}=2000$ MPa leads to a good agreement between the experimental and simulated number of twins.

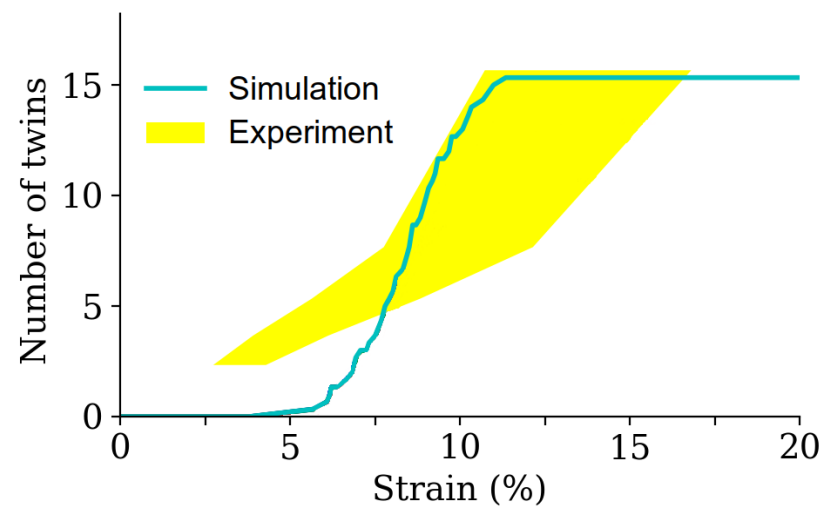

Figure 7: Number of twins as a function of the strain.

The correspondence between the experimental and simulated orientation of the twin planes show that the choice of the active twin system [310] (130) in the simulations is correct. After $14 \%$ strain, about 15 twins are observed in both simulation and experiment.

The clustering of the twin bands observed in the experiment, i.e. the smaller spacing between the twins on the left part of the representative volume in Fig. 6 is slightly different from the more homogeneous twin distribution reproduced by the simulations. This is because a constant interaction length scale $l_{0}$ is used through the entire domain. Variability of the length scale $l_{0}$ could be included to represent the different possible dislocation-twin configurations. This is because dislocation lines with different geometries and belonging to different slip planes would interact 
differently with the twin interface. In the present model, only straight dislocation lines interacting with a twin interface are considered.

\subsection{Dependence on the local twin-cale dislocations interaction coefficient $k_{\beta}$}

A large uncertainty is present in the determination of the initial GND density due to the relatively coarse step $(0.5 \mu \mathrm{m})$ used in the EBSD scan. Therefore, a parametric study of the variable $k_{\beta}$ in Eq. (25) is needed.

The twin phase field for three different values of the product $k_{\beta} \mu_{\beta} b_{\beta} b_{\alpha}$ at $9 \%$ strain is shown in Fig. 8. All three cases show asymmetry in twin nucleation. However, if the product $k_{\beta} \mu_{\beta} b_{\beta} b_{\alpha}$ is increased to $1.5 \mathrm{MPa} \mu \mathrm{m}^{2}$, twin nucleation does not take place in the top-right part and in the left part of the representative volume, corresponding to the location of the low angle boundary (region 1). The simulated number of twins decreases linearly in the three Figures 8 (a), (b) and (c).

This parametric study confirms that the asymmetry in the twin nucleation observed in Fig. 6 is caused by the initial GND density. Because of the sharp difference in the simulated twin phase field when the product $k_{\beta} \mu_{\beta} b_{\beta} b_{\alpha}$ is increased from $0.5 \mathrm{MPa} \mu \mathrm{m}^{2}$ to $1.5 \mathrm{MPa} \mu \mathrm{m}^{2}$, the comparison between the simulated and experimental results allows for a precise determination of the product $k_{\beta} \mu_{\beta} b_{\beta} b_{\alpha}$. The uncertainty in $k_{\beta}$ is therefore linked to the uncertainty in the initial GND density.

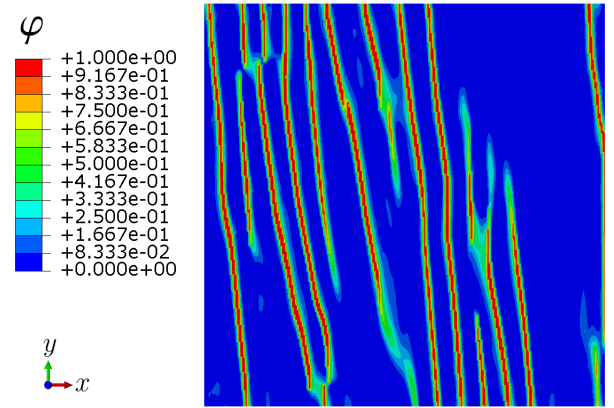

(a) $k_{\beta} \mu_{\beta} b_{\beta} b_{\alpha}=0.5 \mathrm{MPa} \mu \mathrm{m}^{2}$

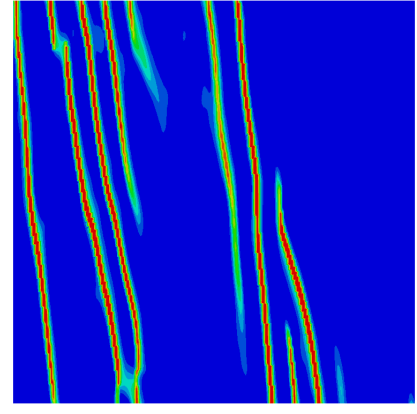

(b) $k_{\beta} \mu_{\beta} b_{\beta} b_{\alpha}=1.0 \mathrm{MPa} \mu \mathrm{m}^{2}$

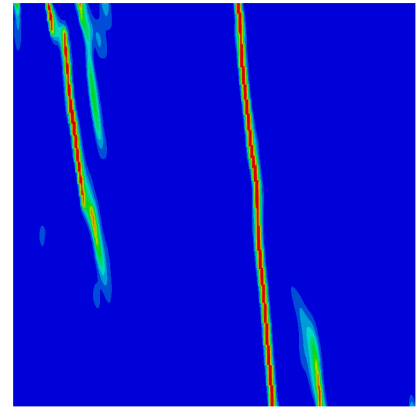

(c) $k_{\beta} \mu_{\beta} b_{\beta} b_{\alpha}=1.5 \mathrm{MPa} \mu \mathrm{m}^{2}$

Figure 8: Twin phase field after 9\% strain. Dependence on the local twin-dislocations interaction coefficient $k_{\beta}$.

\section{Discussion}

The mechanism for twin growth that the simulations in Fig. 6 suggest is described in the following. Initial twin nucleation takes place far from pre-existing dislocations. Once a twin is completely nucleated, the interaction between residual dislocations at the twin interface and mobile dislocations that can cause twin growth is present, as shown in Fig. 2 and described by Eq. (24). After the twin has reached a certain thickness, this interaction prevents the further growth of the twin in a direction parallel to the twin plane normal.

The calibrated value of the length scale $l_{0}=10 \mu \mathrm{m}$ is characteristic of the long range interaction between dislocations [75]. The calibrated value of the prefactor $\tau_{\text {twin }}^{0}=2000 \mathrm{MPa}$ has the following physical interpretation: after $14 \%$ strain, the twin volume fraction in the region of interest is about $20 \%$. Therefore, given a point near an existing twin, the average twin phase field in the 
cylindrical integration region in Eq. (24) is approximately 0.2 . The contribution of the non-local term $\tau_{\text {twin }}(\varphi)$ to the CRSS for twinning is therefore about $400 \mathrm{MPa}$, according to Eq. (24). This is enough to stop twin growth and to favour slip as the dominant plastic deformation mechanism, given the characteristic values of the CRSS for slip, reported in Table 3. The parameter $\tau_{\text {twin }}^{0}$ represents the interaction strength between mobile dislocations and residual dislocations at the twin interface, as described in Section 2.4.

The strong dependence of the twin volume fraction, which is measurable by EBSD, at a given strain on the local twin-dislocations interaction coefficient $k_{\beta}$, as shown in Fig. 8, indicates that CPFE simulations at the micrometre length scale can precisely determine $k_{\beta}$ if the dislocation density is known with high accuracy. Therefore, measurements based on cross correlation methods and direct dislocation imaging will be needed in future studies [63].

The initial nucleation of twins far from pre-existing dislocations is remarkable because most mechanisms proposed in the literature for dislocation nucleation require the presence of initial dislocations. These can be individual dislocations on a cross slip plane with respect to the twinning partials [10], such as in the pole mechanism proposed for low stacking fault energy (SFE) FCC metals [13], or pairs of coplanar dislocations with different Burgers vectors, such as in the fault pair model [12]. Moreover, the stress concentration due to dislocation pile-ups is believed to be necessary to facilitate some of these mechanisms [29].

The present comparison between CPFE simulations and EBSD experiments does not exclude the above mentioned mechanisms because twin nucleation can take place outside of the imaged region of interest. However, it clearly shows that the presence of concentrations of pre-existing dislocations can prevent the nucleation and propagation of twinning partials. These mechanisms are favoured in low dislocation density regions and dislocation pile-ups may not play a role.

The present study shows that the linear relationship between the critical stress for twin growth and the total dislocation density in Eq. (25) can be applied at a length scale smaller than the twin thickness and not only for homogenised twin models [74].

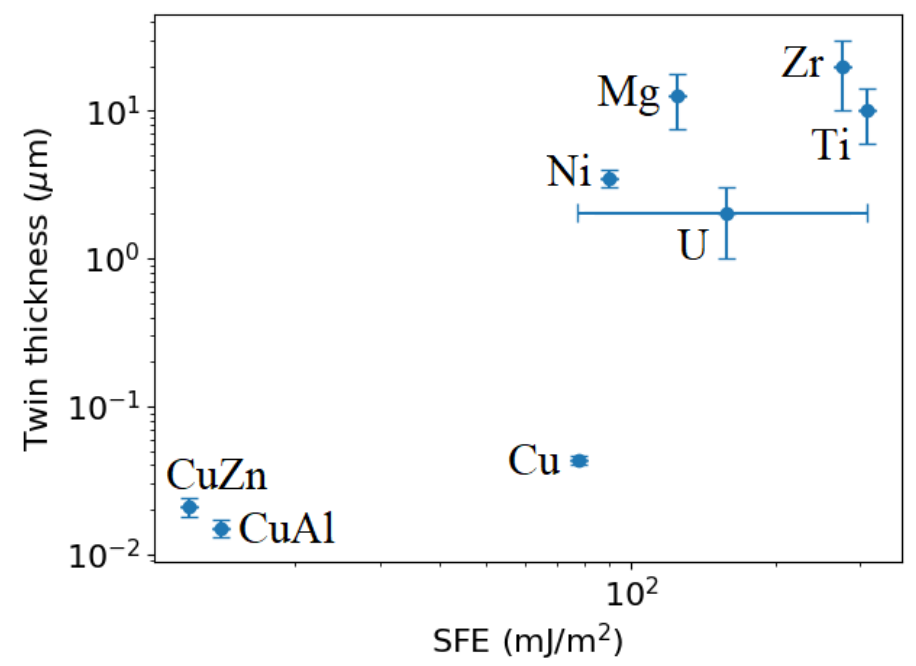

Figure 9: Twin thickness as a function of the SFE: $\mathrm{CuZn}, \mathrm{CuAl}, \mathrm{Cu}$ [25], $\mathrm{Ni}[85], \mathrm{Mg}[86,87], \mathrm{U}$ [88, 89], $\mathrm{Zr}$ [90, 91], Ti $[92,28]$. 
Even though the discrete twin model has been calibrated for $\alpha$-uranium, it can be applied to a wider range of metals, thanks to the physical interpretation of the parameters previously described. The parameter $\tau_{\beta}^{0}$, associated with twin nucleation, is proportional to the SFE $\gamma$. The parameter $\tau_{\text {twin }}^{0}$ is not strongly dependent on the SFE because it is associated with the interaction between mobile dislocations and residual dislocations at the twin interface, which is a long range interaction with a characteristic distance $l_{0}=10 \mu \mathrm{m}$. Indeed, this length scale is much larger than the distance between partials in a dissociated dislocation [93].

Therefore, the ratio $\tau_{\beta}^{0} / \tau_{\text {twin }}^{0}$ is proportional to the SFE. From the results shown in Section 3.2, the model predicts that the smaller $\tau_{\text {twin }}^{0}$ (larger SFE), the larger is the twin thickness after a given strain. The model prediction in Fig. 4 (a), for $\tau_{\text {twin }}^{0}=50 \mathrm{MPa}$, shows that a critical value of the SFE exists above which a single large twin forms during deformation instead of a pattern constituted of several micron-sized twins. This model prediction is in agreement with experimental observation of twins in $\mathrm{Cu}-\mathrm{Zn}$ and $\mathrm{Cu}-\mathrm{Al}$ alloys, in which the stacking fault energy decreases with the addition of alloying elements [94]. A direct proportionality between twin thickness and SFE has been demonstrated using transmission electron microscopy [25].

However, systematic studies of the twin thickness for different metals deformed with the same strain amplitude are not available. The present experimental results on $\alpha$-uranium (Fig. 6) suggest that the average thickness of the micron-sized twins does not strongly depend on the applied strain. Therefore, a literature survey has been carried out to determine the observed twin thickness for different metals, which varies in different samples and from grain to grain, as a function of the SFE. The results are shown in Figure 9, in which the error bars represent the variability of the observed twin thickness. Among the materials reviewed in the literature, $U$ is the one with the largest uncertainty in the SFE. The correlation found is consistent with the model predictions: lower SFE is associated with lower twin thickness.

\section{Conclusions}

A phase field model to describe the nucleation and growth of discrete twins in $\alpha$-uranium has been developed. It is implemented in a CPFE solver and coupled with a dislocation-based constitutive model for slip.

The model for twinning is based on two main parameters: $\tau_{\beta}^{0}$, controlling the twin nucleation, and $\tau_{\text {twin }}^{0}$, controlling the twin growth. These are calibrated using in-situ EBSD experiments on $\alpha$-uranium. Specifically, the number of twins as a function of the strain and their thickness in a region of interest is reproduced.

The nucleation parameter $\tau_{\beta}^{0}$ is interpreted as the RSS to activate possible nucleation mechanisms predicted by atomistic simulations [10]. It is directly proportional to the SFE of the metal considered.

Twin growth is modelled by introducing a non-local term to describe the repulsive stress applied by residual dislocations at the twin interface on incoming dislocations, which can interact with the twin interface and increase the twin thickness by one layer. When the twin thickness and the density of residual dislocations have reached a critical value, further twin growth is prevented. The agreement between simulations and experiment shows that this mechanism is sufficient to limit twin growth and can explain the observed twin thickness. 
The effect of pre-existing dislocations on twin nucleation and growth has been investigated. The model is able to explain the preferential nucleation and growth of twins in regions in which the initial dislocation density is lower. The additional hardening that dislocations contribute to the twin system can be quantified at a length scale that is smaller than the twin thickness.

This paper shows that the comparison between CPFE simulations, which are able to calculate the local stress field, and in-situ EBSD experiments can be employed to understand the physical mechanisms responsible for twinning. The model predicts that materials with a large SFE produces thicker twins for a prescribed strain. Thus, the approach can be used to simulate twinning in a wide range of materials.

\section{Acknowledgements}

The authors acknowledge financial support from AWE plc for this research, program manager: Dr John Askew. ET acknowledges support from the Engineering and Physical Sciences Research Council under Fellowship grant EP/N007239/1.

\section{Appendix A. Derivation of the resolved shear stress}

The resolved shear stress used in section 2.1 [54]:

$$
\tau_{\alpha}=\boldsymbol{F}_{e}^{T} \boldsymbol{F}_{e} \mathbf{S}:\left(\boldsymbol{s}_{\alpha} \otimes \boldsymbol{n}_{\alpha}\right),
$$

can be expressed as a function of the Cauchy stress $\boldsymbol{\sigma}$ using the second P-K stress $\mathbf{S}=J_{e} \boldsymbol{F}_{e}^{-1} \boldsymbol{\sigma} \boldsymbol{F}_{e}^{-T}$ [95]:

$$
\tau_{\alpha}=J_{e} \boldsymbol{F}_{e}^{T} \boldsymbol{\sigma} \boldsymbol{F}_{e}^{-T}:\left(\boldsymbol{s}_{\alpha} \otimes \boldsymbol{n}_{\alpha}\right)=\boldsymbol{\sigma}:\left(\boldsymbol{F}_{e} \boldsymbol{s}_{\alpha} \otimes J_{e} \boldsymbol{F}_{e}^{-T} \boldsymbol{n}_{\alpha}\right) .
$$

$\boldsymbol{F}_{e} \boldsymbol{s}_{\alpha}$ and $J_{e} \boldsymbol{F}_{e}^{-T} \boldsymbol{n}_{\alpha}$ (Nanson's relation) are the slip direction and slip plane normal in the current configuration [96]. The difference between the transformation law for the slip direction and slip plane normal is due to the fact that the slip direction is a vector, while the slip plane normal is a pseudovector (Nanson's relation). Assuming small elastic stretch, Eq. (A.2) corresponds to the standard definition of resolved shear stress [97].

\section{Appendix B. Derivation of the partial derivatives of the elastic free energy}

Using the following chain rule, the derivative of Eq. (8) with respect to the phase field $\varphi$ can be found [40]:

$$
\delta_{\varphi} \Psi_{\mathrm{e}}=\frac{\partial \Psi_{\mathrm{e}}}{\partial \boldsymbol{F}_{e}} \cdot \frac{\partial \boldsymbol{F}_{e}}{\partial \boldsymbol{F}_{p}^{-1}} \cdot \frac{\partial \boldsymbol{F}_{p}^{-1}}{\partial \boldsymbol{L}_{p}} \cdot \frac{\partial \boldsymbol{L}_{p}}{\partial \varphi} \delta \varphi .
$$

The following identities hold [55]:

$$
\frac{\partial \Psi_{\mathrm{e}}}{\partial \mathbf{E}_{e}}=\frac{1}{2} \frac{\partial}{\partial \mathbf{E}_{e}}\left(\mathbf{E}_{e}: \mathbb{C}: \mathbf{E}_{e}\right)=\mathbb{C}: \mathbf{E}_{e}=\mathbf{S},
$$




$$
\begin{aligned}
{\left[\frac{\partial \mathbf{E}_{e}}{\partial \boldsymbol{F}_{e}}\right]_{i j k l} } & =\frac{1}{2}\left[\frac{\partial}{\partial \boldsymbol{F}_{e}}\right]_{k l}\left[\boldsymbol{F}_{e}^{T} \boldsymbol{F}_{e}-\mathbf{I}\right]_{i j}=\frac{1}{2}\left[\frac{\partial}{\partial \boldsymbol{F}_{e}}\right]_{k l}\left[\boldsymbol{F}_{e}\right]_{m i}\left[\boldsymbol{F}_{e}\right]_{m j}= \\
& =\frac{1}{2}\left(\delta_{i l} \delta_{m k}\left[\boldsymbol{F}_{e}\right]_{m j}+\delta_{k m} \delta_{j l}\left[\boldsymbol{F}_{e}\right]_{m i}\right)=\frac{1}{2}\left(\delta_{i l}\left[\boldsymbol{F}_{e}\right]_{k j}+\delta_{j l}\left[\boldsymbol{F}_{e}\right]_{k i}\right),
\end{aligned}
$$

using (B.2) and (B.3) gives:

$$
\begin{gathered}
\frac{\partial \Psi_{\mathrm{e}}}{\partial \boldsymbol{F}_{e}}=\left[\frac{\partial \Psi_{\mathrm{e}}}{\partial \mathbf{E}_{e}}: \frac{\partial \mathbf{E}_{e}}{\partial \boldsymbol{F}_{e}}\right]_{k l}=[\mathbf{S}]_{i j}\left[\frac{\partial \mathbf{E}_{e}}{\partial \boldsymbol{F}_{e}}\right]_{i j k l}= \\
=\frac{1}{2}\left([\mathbf{S}]_{i j} \delta_{i l}\left[\boldsymbol{F}_{e}\right]_{k j}+[\mathbf{S}]_{i j} \delta_{j l}\left[\boldsymbol{F}_{e}\right]_{k i}\right)= \\
=\frac{1}{2}\left(\left[\boldsymbol{F}_{e}\right]_{k j}[\mathbf{S}]_{j l}+\left[\boldsymbol{F}_{e}\right]_{k i}[\mathbf{S}]_{i l}\right)=\boldsymbol{F}_{e} \mathbf{S} \\
{\left[\frac{\partial \boldsymbol{F}_{e}}{\partial \boldsymbol{F}_{p}^{-1}}\right]_{i j k l}=\left[\frac{\partial}{\partial \boldsymbol{F}_{p}^{-1}}\right]_{k l}\left[\boldsymbol{F} \boldsymbol{F}_{p}^{-1}\right]_{i j}=[\boldsymbol{F}]_{i k} \delta_{j l},} \\
\frac{\partial \Psi_{\mathrm{e}}}{\partial \boldsymbol{F}_{p}^{-1}=}\left[\frac{\partial \Psi_{\mathrm{e}}}{\partial \boldsymbol{F}_{e}}: \frac{\partial \boldsymbol{F}_{e}}{\partial \boldsymbol{F}_{p}^{-1}}\right]_{k l}=\left[\boldsymbol{F}_{e} \mathbf{S}\right]_{i j}[\boldsymbol{F}]_{i k} \delta_{j l}=\left[\boldsymbol{F}_{e}\right]_{i m}[\mathbf{S}]_{m l}[\boldsymbol{F}]_{i k}=\boldsymbol{F}^{T} \boldsymbol{F}_{e} \mathbf{S} \\
\boldsymbol{L}_{p}(t+\Delta t)=\dot{\boldsymbol{F}}_{p} \boldsymbol{F}_{p}^{-1}(t+\Delta t) \Longrightarrow \boldsymbol{L}_{p}(t+\Delta t) \Delta t=\mathbf{I}-\boldsymbol{F}_{p}(t) \boldsymbol{F}_{p}^{-1}(t+\Delta t) \\
\boldsymbol{F}_{p}^{-1}(t+\Delta t)=\boldsymbol{F}_{p}^{-1}(t)\left(\mathbf{I}-\boldsymbol{L}_{p}(t+\Delta t) \Delta t\right) \Longrightarrow \frac{1}{\Delta t}\left[\left.\frac{\partial \boldsymbol{F}_{p}^{-1}}{\partial \boldsymbol{L}_{p}}\right|_{t+\Delta t}\right]_{i j k l}=-\left[\boldsymbol{F}_{p}^{-1}(t)\right]_{i k} \delta_{j l}
\end{gathered}
$$

Combining (B.6) and (B.7) gives

$$
\frac{1}{\Delta t} \frac{\partial \Psi_{\mathrm{e}}}{\partial \boldsymbol{L}_{p}}=\frac{1}{\Delta t}\left[\frac{\partial \Psi_{\mathrm{e}}}{\partial \boldsymbol{F}_{p}^{-1}}: \frac{\partial \boldsymbol{F}_{p}^{-1}}{\partial \boldsymbol{L}_{p}}\right]_{k l}=-\left[\boldsymbol{F}^{T} \boldsymbol{F}_{e} \mathbf{S}\right]_{i j}\left[\boldsymbol{F}_{p}^{-1}\right]_{i k} \delta_{j l}=-\left[\boldsymbol{F}_{p}^{-T} \boldsymbol{F}^{T} \boldsymbol{F}_{e} \boldsymbol{S}\right]_{k l}=-\boldsymbol{F}_{e}^{T} \boldsymbol{F}_{e} \boldsymbol{S},
$$

as $\boldsymbol{F}_{p}^{-T}=\boldsymbol{F}_{e}^{T} \boldsymbol{F}^{-T}$, which follows from (1). Differentiating equation (2) gives:

$$
\begin{gathered}
\Delta t \frac{\partial \boldsymbol{L}_{p}}{\partial \varphi}=\gamma_{\beta}^{\mathrm{twin}} \boldsymbol{s}_{\beta} \otimes \boldsymbol{n}_{\beta}, \\
\frac{\partial \Psi_{e}}{\partial \varphi}=\frac{\partial \Psi_{e}}{\partial \boldsymbol{L}_{p}}: \frac{\partial \boldsymbol{L}_{p}}{\partial \varphi}=-\gamma_{\beta}^{\mathrm{twin}} \boldsymbol{F}_{e}^{T} \boldsymbol{F}_{e} \boldsymbol{S}:\left(\boldsymbol{s}_{\beta} \otimes \boldsymbol{n}_{\beta}\right)=-\gamma_{\beta}^{\mathrm{twin}} \tau_{\beta},
\end{gathered}
$$

where summation over repeated indices is assumed and $\delta_{i j}$ is the Kronecker delta. The partial derivatives are calculated at constant displacement field $\mathbf{u}$ and, therefore, constant deformation gradient $\boldsymbol{F}$. Therefore, in Eq. (B.5), $\boldsymbol{F}$ is treated as a constant. The partial derivatives are calculated with respect to the phase field at the current time $\varphi(t+\Delta t)$, therefore $\boldsymbol{F}_{p}^{-1}(t)$ is treated as a constant in (B.7). For the same reason, in Eq. (B.7), $\boldsymbol{L}_{p}(t+\Delta t)$ at the current time is used and the relationship with $\boldsymbol{F}_{p}(t+\Delta t)$ at the current time is found from Eq. (2). Since $\boldsymbol{L}_{p}$ depends on the twin direction and normal at the current time, $\boldsymbol{s}_{\beta}$ and $\boldsymbol{n}_{\beta}$ are calculated in the current configuration. 


\section{References}

[1] J. Christian, S. Mahajan, Deformation twinning, Progress in Materials Science 39 (1995) 1 - 157.

[2] R. W. Cahn, Twinning and slip in $\alpha$-uranium, Acta Crystallographica 4 (1951) 470.

[3] J. P. Hirth, J. Lothe, Theory of dislocations, Krieger Pub. Co, 2nd edition, 1982.

[4] M. Sauzay, M. O. Moussa, Prediction of grain boundary stress fields and microcrack initiation induced by slip band impingement, International Journal of Fracture 184 (2013) $215-240$.

[5] Y. Guo, H. Abdolvand, T. Britton, A. Wilkinson, Growth of $\{11 \overline{2} 2\}$ twins in titanium: A combined experimental and modelling investigation of the local state of deformation, Acta Materialia 126 (2017) $221-235$.

[6] S. B. Biner, J. R. Morris, The effects of grain size and dislocation source density on the strengthening behaviour of polycrystals: a two-dimensional discrete dislocation simulation, Philosophical Magazine 83 (2003) 36773690.

[7] H. Fan, S. Aubry, A. Arsenlis, J. A. El-Awady, The role of twinning deformation on the hardening response of polycrystalline magnesium from discrete dislocation dynamics simulations, Acta Materialia 92 (2015) $126-$ 139.

[8] H. Mirkhani, S. P. Joshi, Mechanism-based crystal plasticity modeling of twin boundary migration in nanotwinned face-centered-cubic metals, Journal of the Mechanics and Physics of Solids 68 (2014) $107-133$.

[9] Q. Li, S. Xue, J. Wang, S. Shao, A. H. Kwong, A. Giwa, Z. Fan, Y. Liu, Z. Qi, J. Ding, H. Wang, J. R. Greer, H. Wang, X. Zhang, High-strength nanotwinned Al alloys with 9R phase, Advanced Materials 30 (2018) 1704629.

[10] P. Chowdhury, H. Sehitoglu, Atomistic Energetics and Critical Twinning Stress Prediction in Face and Body Centered Cubic Metals: Recent Progress, Journal of engineering materials and technology-transactions of the ASME 140 (2018).

[11] J. A. Venables, Deformation twinning in face-centred cubic metals, The Philosophical Magazine: A Journal of Theoretical Experimental and Applied Physics 6 (1961) 379-396.

[12] S. Mahajan, G. Chin, Formation of deformation twins in f.c.c. crystals, Acta Metallurgica 21 (1973) 1353 1363.

[13] A. W. Sleeswyk, Perfect dislocation pole models for twinning in the f.c.c. and b.c.c lattices, The Philosophical Magazine: A Journal of Theoretical Experimental and Applied Physics 29 (1974) 407-421.

[14] S. Ogata, J. Li, S. Yip, Energy landscape of deformation twinning in bec and fcc metals, Phys. Rev. B 71 (2005) 224102.

[15] L. Capolungo, I. J. Beyerlein, Nucleation and stability of twins in hcp metals, Phys. Rev. B 78 (2008) 024117.

[16] A. Cottrell, B. Bilby, Lx. a mechanism for the growth of deformation twins in crystals, The London, Edinburgh, and Dublin Philosophical Magazine and Journal of Science 42 (1951) 573-581.

[17] Z. Jin, T. R. Bieler, An in-situ observation of mechanical twin nucleation and propagation in tial, Philosophical Magazine A 71 (1995) 925-947.

[18] A. Ojha, H. Sehitoglu, L. Patriarca, H. Maier, Twin migration in Fe-based bcc crystals: theory and experiments, Philosophical Magazine 94 (2014) 1816-1840.

[19] X. Zhao, C. Lu, A. K. Tieu, L. Zhan, L. Pei, M. Huang, Deformation mechanisms and slip-twin interactions in nanotwinned body-centered cubic iron by molecular dynamics simulations, Computational Materials Science 147 (2018) $34-48$.

[20] M. Gussev, P. Edmondson, K. Leonard, Beam current effect as a potential challenge in SEM-EBSD in situ tensile testing, Materials Characterization 146 (2018) 25 - 34.

[21] I. Beyerlein, C. Tomé, A dislocation-based constitutive law for pure $\mathrm{Zr}$ including temperature effects, International Journal of Plasticity 24 (2008) $867-895$.

[22] M. Ardeljan, I. J. Beyerlein, M. Knezevic, Effect of dislocation density-twin interactions on twin growth in AZ31 as revealed by explicit crystal plasticity finite element modeling, International Journal of Plasticity 99 (2017) $81-101$.

[23] A. A. Salem, S. R. Kalidindi, R. D. Doherty, Strain hardening of titanium: role of deformation twinning, Acta Materialia 51 (2003) 4225 - 4237.

[24] R. Cahn, Plastic deformation of alpha-uranium; twinning and slip, Acta Metallurgica 1 (1953) 49 - 70. 
[25] Y. Zhang, N. Tao, K. Lu, Effect of stacking-fault energy on deformation twin thickness in Cu-Al alloys, Scripta Materialia 60 (2009) $211-213$.

[26] J. Narayan, Y. T. Zhu, Self-thickening, cross-slip deformation twinning model, Applied Physics Letters 92 (2008) 151908.

[27] F. Fischer, F. Appel, H. Clemens, A thermodynamical model for the nucleation of mechanical twins in TiAl, Acta Materialia 51 (2003) 1249 - 1260.

[28] A. Ghaderi, M. R. Barnett, Sensitivity of deformation twinning to grain size in titanium and magnesium, Acta Materialia 59 (2011) $7824-7839$.

[29] J. Cohen, J. Weertman, A dislocation model for twinning in f.c.c. metals, Acta Metallurgica 11 (1963) 996 998.

[30] M. Meyers, O. Vöhringer, V. Lubarda, The onset of twinning in metals: a constitutive description, Acta Materialia 49 (2001) 4025 - 4039.

[31] M. Homayonifar, J. Mosler, Efficient modeling of microstructure evolution in magnesium by energy minimization, International Journal of Plasticity 28 (2012) 1 - 20.

[32] H. Abdolvand, M. R. Daymond, Multi-scale modeling and experimental study of twin inception and propagation in hexagonal close-packed materials using a crystal plasticity finite element approach — part i: Average behavior, Journal of the Mechanics and Physics of Solids 61 (2013) 783 - 802.

[33] H. Abdolvand, M. R. Daymond, Multi-scale modeling and experimental study of twin inception and propagation in hexagonal close-packed materials using a crystal plasticity finite element approach; part ii: Local behavior, Journal of the Mechanics and Physics of Solids 61 (2013) $803-818$.

[34] M. Kasemer, P. Dawson, A finite element methodology to incorporate kinematic activation of discrete deformation twins in a crystal plasticity framework, Computer Methods in Applied Mechanics and Engineering 358 (2020) 112653.

[35] M. Ardeljan, M. Knezevic, T. Nizolek, I. J. Beyerlein, N. A. Mara, T. M. Pollock, A study of microstructuredriven strain localizations in two-phase polycrystalline hcp/bcc composites using a multi-scale model, International Journal of Plasticity 74 (2015) $35-57$.

[36] J. Clayton, J. Knap, A phase field model of deformation twinning: Nonlinear theory and numerical simulations, Physica D: Nonlinear Phenomena 240 (2011) 841 - 858.

[37] H. Qiao, M. Barnett, P. Wu, Modeling of twin formation, propagation and growth in a Mg single crystal based on crystal plasticity finite element method, International Journal of Plasticity 86 (2016) 70 - 92.

[38] R. Jeyaraam, V. S. Sarma], S. Vedantam, Phase field modelling of annealing twin formation, evolution and interactions during grain growth, Computational Materials Science 182 (2020) 109787.

[39] S. Hu, C. H. Henager, L. Chen, Simulations of stress-induced twinning and de-twinning: A phase field model, Acta Materialia 58 (2010) 6554 - 6564.

[40] C. Liu, P. Shanthraj, M. Diehl, F. Roters, S. Dong, J. Dong, W. Ding, D. Raabe, An integrated crystal plasticity phase field model for spatially resolved twin nucleation, propagation, and growth in hexagonal materials, International Journal of Plasticity 106 (2018) 203 - 227.

[41] C. Liu, P. Shanthraj, J. Robson, M. Diehl, S. Dong, J. Dong, W. Ding, D. Raabe, On the interaction of precipitates and tensile twins in magnesium alloys, Acta Materialia 178 (2019) 146 - 162.

[42] N. Grilli, K. G. Janssens, H. V. Swygenhoven, Crystal plasticity finite element modelling of low cycle fatigue in fcc metals, Journal of the Mechanics and Physics of Solids 84 (2015) $424-435$.

[43] S. Sandfeld, M. Zaiser, Pattern formation in a minimal model of continuum dislocation plasticity, Modelling and Simulation in Materials Science and Engineering 23 (2015) 065005.

[44] C. A. Duarte, N. Grilli, M. Koslowski, Effect of initial damage variability on hot-spot nucleation in energetic materials, Journal of Applied Physics 124 (2018) 025104.

[45] F. Roters, M. Diehl, P. Shanthraj, P. Eisenlohr, C. Reuber, S. Wong, T. Maiti, A. Ebrahimi, T. Hochrainer, H.-O. Fabritius, S. Nikolov, M. Friák, N. Fujita, N. Grilli, K. Janssens, N. Jia, P. Kok, D. Ma, F. Meier, E. Werner, M. Stricker, D. Weygand, D. Raabe, DAMASK - the Düsseldorf advanced material simulation kit for modeling multi-physics crystal plasticity, thermal, and damage phenomena from the single crystal up to the component scale, Computational Materials Science (2018).

[46] F. Dunne, D. Rugg, A. Walker, Lengthscale-dependent, elastically anisotropic, physically-based hcp crystal 
plasticity: Application to cold-dwell fatigue in ti alloys, International Journal of Plasticity 23 (2007) 1061 1083.

[47] N. Grilli, E. Tarleton, P. D. Edmondson, M. N. Gussev, A. C. F. Cocks, In situ measurement and modelling of the growth and length scale of twins in $\alpha$-uranium, Phys. Rev. Materials 4 (2020) 043605.

[48] F. Roters, P. Eisenlohr, C. Kords, D. Tjahjanto, M. Diehl, D. Raabe, Damask: the Düsseldorf advanced material simulation kit for studying crystal plasticity using an fe based or a spectral numerical solver, Procedia IUTAM 3 (2012) 3 - 10. IUTAM Symposium on Linking Scales in Computations: From Microstructure to Macro-scale Properties.

[49] S. R. Kalidindi, Incorporation of deformation twinning in crystal plasticity models, Journal of the Mechanics and Physics of Solids 46 (1998) $267-290$.

[50] F. Roters, P. Eisenlohr, L. Hantcherli, D. Tjahjanto, T. Bieler, D. Raabe, Overview of constitutive laws, kinematics, homogenization and multiscale methods in crystal plasticity finite-element modeling: Theory, experiments, applications, Acta Materialia 58 (2010) 1152 - 1211.

[51] P. Zhou, D. Xiao, W. Wang, G. Sang, Y. Zhao, D. Zou, L. He, Twinning behavior of polycrystalline alphauranium under quasi static compression, Journal of Nuclear Materials 478 (2016) $83-90$.

[52] R. Asaro, A. Needleman, Overview no. 42 texture development and strain hardening in rate dependent polycrystals, Acta Metallurgica 33 (1985) 923 - 953.

[53] N. Grilli, M. Koslowski, The effect of crystal orientation on shock loading of single crystal energetic materials, Computational Materials Science 155 (2018) 235 - 245.

[54] M. Jafari, M. Jamshidian, S. Ziaei-Rad, D. Raabe, F. Roters, Constitutive modeling of strain induced grain boundary migration via coupling crystal plasticity and phase-field methods, International Journal of Plasticity 99 (2017) $19-42$.

[55] F. Roters, Advanced material models for the crystal plasticity finite element method - Development of a general CPFEM framework, RWTH Aachen, Germany.

[56] R. Madec, B. Devincre, L. P. Kubin, From dislocation junctions to forest hardening, Phys. Rev. Lett. 89 (2002) 255508.

[57] L. Capolungo, I. Beyerlein, G. Kaschner, C. Tomé, On the interaction between slip dislocations and twins in HCP Zr, Materials Science and Engineering: A 513-514 (2009) $42-51$.

[58] N. Grilli, A. C. Cocks, E. Tarleton, Crystal plasticity finite element modelling of coarse-grained $\alpha$-uranium, Computational Materials Science 171 (2020) 109276.

[59] N. Grilli, A. Cocks, E. Tarleton, Crystal plasticity finite element simulations of cast $\alpha$-uranium, in: E. Onate, D. Owen, D. Peric, M. Chiumenti (Eds.), Computational plasticity XV: fundamentals and applications. 15th International Conference on Computational Plasticity - Fundamentals and Applications (COMPLAS), Barcelona, Spain, Sep 03-05, 2019.

[60] M. Knezevic, R. J. McCabe, C. N. Tomé, R. A. Lebensohn, S. R. Chen, C. M. Cady, G. T. G. III, B. Mihaila, Modeling mechanical response and texture evolution of $\alpha$-uranium as a function of strain rate and temperature using polycrystal plasticity, International Journal of Plasticity 43 (2013) $70-84$.

[61] R. McCabe, L. Capolungo, P. Marshall, C. Cady, C. Tomé, Deformation of wrought uranium: Experiments and modeling, Acta Materialia 58 (2010) 5447 - 5459.

[62] U. Kocks, H. Mecking, Physics and phenomenology of strain hardening: the fcc case, Progress in Materials Science 48 (2003) $171-273$.

[63] N. Grilli, K. Janssens, J. Nellessen, S. Sandlöbes, D. Raabe, Multiple slip dislocation patterning in a dislocationbased crystal plasticity finite element method, International Journal of Plasticity 100 (2018) $104-121$.

[64] B. L. Hansen, C. A. Bronkhorst, M. Ortiz, Dislocation subgrain structures and modeling the plastic hardening of metallic single crystals, Modelling and Simulation in Materials Science and Engineering 18 (2010) 055001.

[65] J. D. Clayton, Nonlinear Mechanics of Crystals, Springer, 2011.

[66] M. Calcagnotto, D. Ponge, E. Demir, D. Raabe, Orientation gradients and geometrically necessary dislocations in ultrafine grained dual-phase steels studied by $2 \mathrm{~d}$ and $3 \mathrm{~d}$ ebsd, Materials Science and Engineering: A 527 (2010) $2738-2746$.

[67] A. Irastorza-Landa, N. Grilli, H. V. Swygenhoven, Effect of pre-existing immobile dislocations on the evolution of geometrically necessary dislocations during fatigue, Modelling and Simulation in Materials Science and 
Engineering 25 (2017) 055010.

[68] S. Das, F. Hofmann, E. Tarleton, Consistent determination of geometrically necessary dislocation density from simulations and experiments, International Journal of Plasticity 109 (2018) 18 - 42.

[69] S. A. Kibey, L. L. Wang, J. B. Liu, H. T. Johnson, H. Sehitoglu, D. D. Johnson, Quantitative prediction of twinning stress in fcc alloys: Application to Cu-Al, Phys. Rev. B 79 (2009) 214202.

[70] B. D. Coleman, Thermodynamics of materials with memory, Archive for Rational Mechanics and Analysis 17 (1964) $1-45$.

[71] P. Lynch, M. Kunz, N. Tamura, M. Barnett, Time and spatial resolution of slip and twinning in a grain embedded within a magnesium polycrystal, Acta Materialia 78 (2014) 203 - 212.

[72] P. G. Oberson, S. Ankem, Why twins do not grow at the speed of sound all the time, Physical Review Letters 95 (2005).

[73] M. Ahmed, D. Wexler, G. Casillas, D. G. Savvakin, E. V. Pereloma, Strain rate dependence of deformationinduced transformation and twinning in a metastable titanium alloy, Acta Materialia 104 (2016) $190-200$.

[74] N. Grilli, P. Earp, A. C. Cocks, J. Marrow, E. Tarleton, Characterisation of slip and twin activity using digital image correlation and crystal plasticity finite element simulation: Application to orthorhombic $\alpha$-uranium, Journal of the Mechanics and Physics of Solids 135 (2020) 103800.

[75] D. Hull, D. Bacon, 9 - Dislocation Arrays and Crystal Boundaries, in: D. Hull, D. Bacon (Eds.), Introduction to Dislocations (Fourth Edition), Butterworth-Heinemann, Oxford, fourth edition, 2001, pp. 157 - 192.

[76] A. Irastorza-Landa, N. Grilli, H. V. Swygenhoven, Laue micro-diffraction and crystal plasticity finite element simulations to reveal a vein structure in fatigued cu, Journal of the Mechanics and Physics of Solids 104 (2017) $157-171$.

[77] T. Belytschko, K. Liu, B. Moran, K. Elkhodary, Nonlinear Finite Element Analysis for Continua and Structures, John Wiley \& Sons, New York, 2 edition, 2014.

[78] P. Houtte, Simulation of the rolling and shear texture of brass by the taylor theory adapted for mechanical twinning, Acta Metallurgica 26 (1978) $591-604$.

[79] E. S. Fisher, H. J. McSkimin, Adiabatic elastic moduli of single crystal alpha uranium, Journal of Applied Physics 29 (1958) 1473-1484.

[80] B. Beeler, C. Deo, M. Baskes, M. Okuniewski, First principles calculations of the structure and elastic constants of $\alpha, \beta$ and $\gamma$ uranium, Journal of Nuclear Materials 433 (2013) $143-151$.

[81] C. Calhoun, J. Wollmershauser, D. Brown, R. Mulay, E. Garlea, S. Agnew, Thermal residual strains in depleted $\alpha$-U, Scripta Materialia 69 (2013) 566 - 569.

[82] J. S. Lukesh, Note on the structure of uranium, Acta Crystallographica 2 (1949) 420.

[83] L. Kubin, A. Mortensen, Geometrically necessary dislocations and strain-gradient plasticity: a few critical issues, Scripta Materialia 48 (2003) $119-125$.

[84] R. Hielscher, F. Bartel, T. B. Britton, Gazing at crystal balls: Electron backscatter diffraction pattern analysis and cross correlation on the sphere, Ultramicroscopy 207 (2019) 112836.

[85] Y. Jin, B. Lin, M. Bernacki, G. Rohrer, A. Rollett, N. Bozzolo, Annealing twin development during recrystallization and grain growth in pure nickel, Materials Science and Engineering: A 597 (2014) 295 - 303.

[86] N. R. Kumar, J. Blandin, C. Desrayaud, F. Montheillet, M. Suéry, Grain refinement in az91 magnesium alloy during thermomechanical processing, Materials Science and Engineering: A 359 (2003) 150 - 157.

[87] Y. Liu, P. Z. Tang, M. Y. Gong, R. J. McCabe, J. Wang, C. N. Tomé, Three-dimensional character of the deformation twin in magnesium, Nature Communications 10 (2019) 3308.

[88] I. Saxl, J. Kočík, J. Otruba, Extended dislocations and stacking faults on [110] and (001) planes of $\alpha$-uranium, Journal of Nuclear Materials 25 (1968) $172-178$.

[89] A. Crocker, Stacking faults in alpha uranium, Journal of Nuclear Materials 15 (1965) $121-124$.

[90] S. Hou, H. Lei, Z. Zeng, Hydrogen influence on generalized stacking fault energies of zr 0001 basal plane: a first-principles study, RSC Adv. 6 (2016) 54371-54376.

[91] D. Westlake, Twinning in zirconium, Acta Metallurgica 9 (1961) 327 - 331.

[92] Z. Guo, A. Miodownik, N. Saunders, J.-P. Schillé, Influence of stacking-fault energy on high temperature creep of alpha titanium alloys, Scripta Materialia 54 (2006) $2175-2178$.

[93] D. Hull, D. Bacon, Chapter 5 - Dislocations in Face-centered Cubic Metals, in: D. Hull, D. Bacon (Eds.), 
Introduction to Dislocations (Fifth Edition), Butterworth-Heinemann, Oxford, fifth edition, 2011, pp. 85 - 107.

[94] J. Venables, The electron microscopy of deformation twinning, Journal of Physics and Chemistry of Solids 25 (1964) $685-692$.

[95] N. Grilli, M. Koslowski, The effect of crystal anisotropy and plastic response on the dynamic fracture of energetic materials, Journal of Applied Physics 126 (2019) 155101.

[96] E. de Souza Neto, D. Perić, D. Owen, Computational Methods for Plasticity: Theory and Applications, Wiley, 1st edition, 2008.

[97] D. Hull, D. Bacon, Chapter 3 - Movement of Dislocations, in: D. Hull, D. Bacon (Eds.), Introduction to Dislocations (Fifth Edition), Butterworth-Heinemann, Oxford, fifth edition, 2011, pp. 43 - 62. 